TRANSACTIONS OF THE

AMERICAN MATHEMATICAL SOCIETY

Volume 301, Number 2, June 1987

\title{
PRIME IDEALS IN POLYCYCLIC CROSSED PRODUCTS
}

\author{
D. S. PASSMAN
}

\begin{abstract}
In this paper, we describe the prime ideals $P$ in crossed products $R * G$ with $R$ a right Noetherian ring and with $G$ a polycyclic-by-finite group. This is achieved through a series of reductions. To start with, we may assume that $P \cap R=0$ so that $R$ is a $G$-prime ring. The first step uses a technique of $\mathrm{M}$. Lorenz and the author to reduce to a prime ring and a subgroup of finite index in $G$. Next if $R$ is prime, then we show that the prime ideals of $R * G$ disjoint from $R$ are explicitly determined by the primes of a certain twisted group algebra of a normal subgroup of $G$. Finally the prime ideals in twisted group algebras of polycyclic-by-finite groups are studied by lifting the situation to ordinary group algebras where the results of J. E. Roseblade can be applied.
\end{abstract}

Let $G$ be a multiplicative group, let $R$ be a ring and let $R * G$ be a crossed product of $G$ over $R$. The aim of this paper is to study the prime ideals $P$ of $R * G$ disjoint from $R$, that is with $P \cap R=0$. The main result here describes these primes when $R$ is right Noetherian and $G$ is polycyclic-by-finite.

This description is achieved in a series of steps. First suppose that there is a prime of $R * G$ disjoint from $R$. Then $R$ must be a $G$-prime ring and hence, since $R$ is Noetherian, there exists a minimal prime $Q$ of $R$ with $\bigcap_{x \in G} Q^{x}=0$. If $H$ denotes the stabilizer of $Q$ in $G$, then $|G: H|<\infty$ and, by a modification of the proof in [2], there exists a precisely defined one-to-one correspondence between the primes $P$ of $R * G$ with $P \cap R=0$ and the primes $L$ of $(R / Q) * H$ with $L \cap(R / Q)=0$. This therefore reduces the consideration to prime coefficient rings.

Now let $R$ be prime. In this case, $\Gamma=R * G$ extends uniquely to $\Gamma^{\prime}=S * G$ where $S=Q_{s}(R)$ is the symmetric Martindale ring of quotients of $R$. If $F=\mathbf{Z}(S)$ is the extended centroid of $R$, then $\mathbf{C}_{\Gamma^{\prime}}(S)=F^{t}\left[G_{\text {inn }}\right]$ is a twisted group algebra over $F$ of $G_{\text {inn }}$, the normal subgroup of $G$ consisting of those elements which induce inner automorphisms on $S$. Furthermore there is a precisely described one-to-one correspondence between the prime ideals $P$ of $\Gamma$ with $P \cap R=0$ and the $G$-prime ideals $\tilde{P}$ of $F^{t}\left[G_{\text {inn }}\right]$. Indeed $P=\tilde{P} \Gamma^{\prime} \cap \Gamma$ and the problem is therefore reduced to the consideration of $G$-primes in a twisted group algebra. The argument here is actually an offshoot of recent work $[\mathbf{7}]$ on Lie algebra smash products.

Finally the $G$-primes of $F^{t}\left[G_{\text {inn }}\right]$ are closely related to the $G$-orbital prime ideals of $F^{t}\left[G_{\text {inn }}\right]$. Since $G_{\text {inn }}$ is polycylic-by-finite, the structure of the primes of $F^{t}\left[G_{\text {inn }}\right]$ follows easily from the work of $[8]$ by lifting the group $G_{\text {inn }}$. We therefore obtain a number of corollaries on incomparability and prime length.

Received by the editors February 5, 1986 and, in revised form, May 13, 1986.

1980 Mathematics Subject Classification (1985 Revision). Primary 16A27, 16A12.

Research supported in part by NSF Grant No. MCS82-19678. 
This paper is in essence a combination of ideas and techniques from several different sources. The results obtained extend the finite group theorems of $[\mathbf{1}]$ to polycyclic-by-finite groups under the additional assumption that the coefficient ring is Noetherian.

1. $G$-prime coefficient rings. Let $G$ be a multiplicative group and let $R$ be a ring with 1 . Then a crossed product $R * G$ of $G$ over $R$ is an associative ring containing for each $x \in G$ an element $\bar{x} \in R * G$. The set $\bar{G}=\{\bar{x} \mid x \in G\}$ is a left $R$-basis for $R * G$ so that every element $\alpha$ is uniquely writable as a finite sum

$$
\alpha=\sum_{x \in G} r_{x} \bar{x}
$$

with $r_{x} \in R$. The addition in $R * G$ is the obvious one and the multiplication is given by the formulas

$$
\bar{x} \bar{y}=t(x, y) \overline{x y}, \quad r \bar{x}=\bar{x} r^{\bar{x}}
$$

for all $x, y \in G$ and $r \in R$. Here $t: G \times G \rightarrow U$ is a map from $G \times G$ to the group of units $U=\mathcal{U}(R)$ of $R$. Furthermore for fixed $x \in G$ the map ${ }^{\bar{x}}: r \rightarrow r^{\bar{x}}$ is an automorphism of $R$.

The ring $R * G$ has an identity element, namely $1=t(1,1)^{-1} \overline{1}$ and hence without loss of generality we will assume that $\overline{1}=1$. Moreover each $\bar{x}$ is invertible and in fact

$$
\mathfrak{G}=\{u \bar{x} \mid u \in U, x \in G\}
$$

is a multiplicative group of units of $R * G$, the group of trivial units. Note that $\mathfrak{G}$ acts by conjugation on the naturally embedded subring $R$ of $R * G$. Furthermore $U \triangleleft \mathfrak{G}$ and $\mathfrak{G} / U \simeq G$. Observe that conjugation by $U$ stabilizes all ideals of $R$ and thus there exists a well-defined permutation action of $G \simeq \mathfrak{G} / U$ on the set of these ideals. We say that the $G$-invariant ideal $I$ of $R$ is $G$-prime if for all $G$-stable ideals $A, B$ of $R$ the inclusion $A B \subseteq I$ implies that $A \subseteq I$ or $B \subseteq I$. In particular, $R$ is a $G$-prime ring if $I=0$ is a $G$-prime ideal.

LEMMA 1.1. Let $R * G$ be given.

(i) If $A$ is a $G$-stable ideal of $R$, then

$$
A(R * G)=(R * G) A=A * G \triangleleft R * G
$$

and $(R * G) /(A * G) \simeq(R / A) * G$.

(ii) If $P$ is a prime ideal of $R * G$ then $P \cap R$ is a $G$-prime ideal of $R$.

Proof. (i) It is clear that $A * G$ is an ideal of $R * G$ and that $(R * G) /(A * G)$ is generated by $(R / A)$ and the image of $\bar{G}$. Since the latter is independent over $R / A$, it then follows that $(R * G) /(A * G)$ is a suitable crossed product of $G$ over $R / A$.

(ii) Let $A, B$ be $G$-stable ideals of $R$ with $A B \subseteq P \cap R$. Then

$$
(A * G)(B * G) \subseteq(P \cap R) * G \subseteq P
$$

so the primeness of $P$ yields $A * G \subseteq P$ or $B * G \subseteq P$. Hence either $A$ or $B$ is contained in $P \cap R$.

Recall that a group $G$ is polycylic-by-finite if there exists a finite subnormal series

$$
1=G_{0} \triangleleft G_{1} \triangleleft \cdots \triangleleft G_{n}=G
$$

with each quotient $G_{i+1} / G_{i}$ either infinite cyclic or finite. The following is well known (see for example [5, Theorem 10.2.7]). 
LEMMA 1.2. Let $R * G$ be given with $R$ right Noetherian and with $G$ polycyclicby-finite. Then $R * G$ is right Noetherian.

If $P$ is a prime ideal of $R * G$, then $P \supseteq(P \cap R) * G$ and hence $P /(P \cap R) * G$ is a prime ideal in $(R * G) /(P \cap R) * G \simeq(R / P \cap R) * G$. Since the homomorphism $R * G \rightarrow(R / P \cap R) * G$ is well understood, it suffices to replace $P$ and $R * G$ by their images. Equivalently we can assume that $P \cap R=0$ and hence that $R$ is a $G$-prime ring by Lemma 1.1(ii). In this section we study this situation and show how to reduce the problem to prime coefficient rings. To do this we use the techniques of $[2]$ with some minor changes. To avoid confusion we have opted to include all relevant details here even though there is considerable duplication.

We assume throughout the remainder of this section that $R * G$ is given with $R$ right Noetherian and with $G$ polycyclic-by-finite. Furthermore $R$ is $G$-prime.

LEMMA 1.3. Let $Q$ be a minimal prime of $R$. Then

(i) $\bigcap_{x \in G} Q^{x}=0$ so $R$ is semiprime.

(ii) $\left\{Q^{x} \mid x \in G\right\}$ is the finite set of minimal primes of $R$.

(iii) Let $H$ denote the stabilizer of $Q$ in $G$ and set $N=\operatorname{ann}_{R} Q$. Then $H$ is a subgroup of $G$ of finite index,

$$
N=\bigcap_{x \notin H} Q^{x} \neq 0
$$

and

$$
0=N \bar{x} N=N \cap N^{x}=N \cap Q
$$

for all $x \in G \backslash H$.

(iv) If $A$ is a nonzero ideal of $R$ with $A \subseteq N$, then $\operatorname{ann}_{R} A=Q$.

Proof. (i) Since $R$ is Noetherian there exist just finitely many minimal primes, say $Q=Q_{1}, Q_{2}, \ldots, Q_{n}$, and $\left(\bigcap_{i} Q_{i}\right)^{m}=0$ for some $m$. Clearly $G$ permutes these ideals and we let $A=\bigcap_{x \in G} Q^{x}$ and $B$ equal the intersection of the remaining primes if any. Then $A$ and $B$ are both $G$-stable and $(A B)^{m}=0$ so either $A$ or $B$ is zero. But $B=0$ implies that $Q \supseteq B$ contradicting the fact that $Q$ is a minimal prime of $R$. Thus $A=0$ and part (i) follows. Part (ii) is now also immediate.

(iii) Since $R$ is semiprime, right and left annihilators of two-sided ideals are equal. Thus $N=\operatorname{ann}_{R} Q$ is unambiguously defined and it is clear from (i) that $N \supseteq \bigcap_{x \notin H} Q^{x}$. On the other hand if $x \notin H$ then $Q^{x} \supseteq Q N=0$ and $Q^{x} \nsupseteq Q$ so $Q^{x} \supseteq N$ and we have $N=\bigcap_{x \notin H} Q^{x} \neq 0$. It follows from this formula that if $x \notin H$ then $Q \supseteq N^{x}$ so $N \cap N^{x} \subseteq N \cap Q=0$. Finally $0=N^{x} N=\bar{x}^{-1} N \bar{x} N$ yields $N \bar{x} N=0$ as required.

(iv) If $0 \neq A \subseteq N$ then certainly $Q \subseteq \operatorname{ann}_{R} N \subseteq \operatorname{ann}_{R} A$. On the other hand, $Q \supseteq A \cdot \operatorname{ann}_{R} A$ and $Q \nsupseteq A$ so we obtain $Q \supseteq \operatorname{ann}_{R} A$.

The notation of the preceding lemma will be kept throughout the remainder of this section. Thus $Q$ is a minimal prime of $R, N=\operatorname{ann}_{R} Q$ and $H$ is the stabilizer of $Q$ in $G$. Furthermore we set $M=\sum_{x \in G} N^{x}$ so that $M$ is a nonzero $G$-stable ideal of $R$. If $\alpha=\sum a_{x} \bar{x} \in R * G$ then we let $\operatorname{Supp} \alpha=\left\{x \in G \mid a_{x} \neq 0\right\}$. If $T$ is any subset of $G$ we let

$$
R * T=\{\alpha \in R * G \mid \operatorname{Supp} \alpha \subseteq T\}
$$

In particular $R * H$ is the naturally embedded crossed product over $H$. Part (ii) of the following lemma is a crucial observation; part (i) is needed for its proof. 
LEMMA 1.4. Let $H$ and $N$ be as above.

(i) Let $V$ be a nonzero right $R$-submodule of $N \bar{G}$ and let $T$ be a finite subset of G. Suppose that $V \cap(R * T) \neq 0$ but that $V \cap\left(R * T^{\prime}\right)=0$ for all $T^{\prime}<T$. Then $T$ is contained in a right coset of $H$.

(ii) Let $I$ be an ideal of $R * G$. Then there exists a nonzero $G$-stable ideal $E$ of $R$ (depending on $I$ ) with

$$
E I \subseteq \bar{G} N(I \cap R * H) \bar{G} .
$$

Proof. (i) Fix $s \in T$. By assumption there exists $0 \neq \alpha=\sum a_{t} \bar{t} \in V \cap R * T$. Since $V \subseteq N \bar{G}$ we have $a_{t} \in N$ for all $t \in T$ and the minimality condition on $T$ implies that $a_{t} \neq 0$ for all $t$. Now for any $q \in Q^{s}$ we have

$$
\alpha q=\sum_{t} a_{t} q^{\bar{t}^{-1}} \bar{t} \in V \cap(R * T)
$$

and since $s \in T$, the summand $a_{s} q^{\bar{s}^{-1}} \in N Q=0$. Thus by minimality again $a_{t} q^{\bar{t}^{-1}}=0$ for all $t$ so $a_{t}^{\bar{t}} \in \operatorname{ann}_{R} Q^{s}=N^{s}$. Thus $0 \neq a_{t}^{\bar{t}} \in N^{s} \cap N^{t}=\left(N \cap N^{t s^{-1}}\right)^{s}$ so $t s^{-1} \in H$ by Lemma 1.3 (iii).

(ii) We show first that there exists a nonzero ideal $B$ of $R$ with

$$
B I \subseteq \bar{G} N(I \cap R * H) \bar{G}
$$

and this is clear if $N I=0$. Thus we may suppose that $V=N I \neq 0$. Note that $V$ satisfies the hypothesis of (i) and that $V$ is a right ideal of $R * G$. Let $\tau$ denote the family of all finite subsets $T$ of $G$ such that $V \cap(R * T) \neq 0$ but $V \cap\left(R * T^{\prime}\right)=0$ for all $T^{\prime}<T$. By (i) above, each $T \in \tau$ is contained in a right coset of $H$. For convenience we choose a canonical element $y=y(T) \in T$ for each $T \in \tau$ and we let

$$
A_{T}=\left\{r \in R \mid \text { there exists } \beta=\sum_{t \in T} b_{t} \bar{t} \in V \text { with } r=b_{y}\right\} .
$$

Since $V$ is an $(R, R)$-subbimodule of $N \bar{G}$, it is clear that each $A_{T}$ is a nonzero ideal of $R$ contained in $N$.

For convenience we also arbitrarily linearly order the elements $T \in \tau$. If $S$ is a finite subset of $G$ we can then define $B_{S}=N \cdot \prod_{T \subseteq S} A_{T}$ where the product is taken with the $T$ 's in $T$ in increasing order. By Lemma 1.3(iv) each $B_{S}$ is a nonzero ideal of $R$ contained in $N$. If $\alpha \in V$ with $|\operatorname{Supp} \alpha|=m$ we show by induction on $m$ that $B_{S} \alpha \subseteq N(I \cap R * H) \bar{G}$ where $S=\operatorname{Supp} \alpha$. This is clear for $m=0$ so assume that $m>0$ and that the result is true for all smaller support sizes. Since $0 \neq \alpha \in V$ there exists $T \subseteq S=\operatorname{Supp} \alpha$ with $T \in \tau$. We assume $T$ is largest possible in the ordering of $\tau$ and we set $y=y(T)$.

Let $\alpha=a \bar{y}+\cdots$ and let $d \in A_{T}$. Then by definition there exists $\beta \in V \cap R * T \subseteq$ $V \cap R * S$ with $\beta=d \bar{y}+\cdots$. Since $T \subseteq S$ it follows that

$$
\gamma=d \alpha-\beta a^{\bar{y}} \in V \cap(R * S) .
$$

But the $\bar{y}$ coefficient of $\gamma$ is zero so Supp $\gamma=S^{\prime}$ has size less than $m$. By induction, $B_{S^{\prime}} \gamma \subseteq N(I \cap R * H) \bar{G}$. Furthermore since $T \subseteq H y$, it is clear that $\beta=\left(\beta \bar{y}^{-1}\right) \bar{y} \in$ $(I \cap R * H) \bar{G}$ so since $B_{S^{\prime}} \subseteq N$ we have $B_{S^{\prime}} \beta a^{\bar{y}} \subseteq N(I \cap R * H) \bar{G}$. This all implies that $B_{S^{\prime}} d \alpha \subseteq N(I \cap R * H) \bar{G}$. Note that $y \notin S^{\prime}$ so $T \nsubseteq S^{\prime}$. Thus if $B^{\prime}$ denotes the 
obvious product with $B_{S}=B^{\prime} A_{T}$, then $B_{S^{\prime}} \supseteq B^{\prime}$ so $B^{\prime} d \alpha \subseteq N(I \cap R * H) \bar{G}$. But this holds for all $d \in A_{T}$ so $B_{S} \alpha \subseteq N(I \cap R * H) \bar{G}$ as required.

Now $R * G$ is right Noetherian by Lemma 1.2 so $N I=\sum_{1}^{n} \alpha_{i} R * G$ for suitable $\alpha_{i} \in V$. By the above for each $i$ there exists a nonzero ideal $B_{i} \subseteq N$ with $B_{i} \alpha_{i} \subseteq$ $N(I \cap R * H) \bar{G}$ so letting $B=\bigcap B_{i} \neq 0$ we have $B N I \subseteq N(I \cap R * H) \bar{G}$ since the latter is a right ideal of $R * G$. Again $B N \neq 0$ so we have shown that

$$
E=\{r \in R \mid r I \subseteq \bar{G} N(I \cap R * H) \bar{G}\}
$$

is not zero. But with the extra $\bar{G}$ factor in front, $\bar{G} N(I \cap R * H) \bar{G} \triangleleft R * G$ so it is clear that $E$ must be a $G$-stable ideal of $R$ and the result follows.

If $I \triangleleft R * G$ we define

$$
I_{H}=\{\alpha \in R * H \mid N \alpha \subseteq I\}
$$

and if $L \triangleleft R * H$ we set

$$
L^{G}=\bigcap_{x \in G}(L \bar{G})^{\bar{x}} .
$$

Furthermore let $\operatorname{tr}_{H}: R * G \rightarrow R * H$ denote the natural projection given by

$$
\operatorname{tr}_{H}\left(\sum_{x \in G} r_{x} \bar{x}\right)=\sum_{x \in H} r_{x} \bar{x} .
$$

LEMMA 1.5. With the above notation we have

(i) $I_{H} \triangleleft R * H$ and if $I \cap R=0$ then $I_{H} \cap R=Q$.

(ii) $L^{G}$ is the unique largest two sided ideal of $R * G$ contained in $L \bar{G}$ and if $L \cap R=Q$ then $L^{G} \cap R=0$.

(iii) $L^{G}$ is the unique largest ideal $I$ of $R * G$ with $\operatorname{tr}_{H}(I) \subseteq L$.

ProOF. (i) Since $I \triangleleft R * G$ and $N \triangleleft R$, it follows that $I_{H}$ is a left $R$-module and a right $R * H$-module. Furthermore both $N$ and $I$ are $H$-stable under conjugation so $I_{H}$ is also $H$-stable. This along with the above implies that $I_{H} \triangleleft R * H$. Finally if $I \cap R=0$ then $r \in I_{H} \cap R$ if and only if $N r \subseteq I \cap R=0$ so $I_{H} \cap R=\operatorname{ann}_{R} N=Q$.

(ii) If $I$ is an ideal of $R * G$ contained in $L \bar{G}$, then since $I$ is $G$-invariant we have $I \subseteq \bigcap_{x \in G}(L \bar{G})^{\bar{x}}=L^{G}$. On the other hand, $L^{G}$ is clearly a right $R * G$-module, a left $R$-module and it is $G$-invariant. Thus $L^{G} \triangleleft R * G$. Finally if $L \cap R=Q$, then $L^{G} \cap R \subseteq L \bar{G}$ implies that $L^{G} \cap R \subseteq L \cap R=Q$. But $L^{G} \cap R$ is $G$-stable so $L^{G} \cap R \subseteq \bigcap_{x \in G} Q^{x}=0$.

(iii) Let $I \triangleleft R * G$. If $I \subseteq L \bar{G}$ then clearly $\operatorname{tr}_{H}(I) \subseteq L$. On the other hand if $\operatorname{tr}_{H}(I) \subseteq L$, then $I \subseteq \operatorname{tr}_{H}(\bar{I}) \bar{G} \subseteq L \bar{G}$. Thus the result follows immediately from (ii) above.

It is clear that the maps ${ }^{G}$ and ${ }_{H}$ are monotone. They also have the following multiplicative properties.

LEMMA 1.6. (i) If $I_{1}$ and $I_{2}$ are ideals of $R * G$ then $\left(I_{1}\right)_{H}(N * H)\left(I_{2}\right)_{H} \subseteq$ $\left(I_{1} I_{2}\right)_{H}$.

(ii) If $L_{1}$ and $L_{2}$ are ideals of $R * H$ then $L_{1}{ }^{G} L_{2} G \subseteq\left(L_{1} L_{2}\right)^{G}$.

Proof. (i) By definition $N I_{H} \subseteq I$ so $N\left(I_{1}\right)_{H} \cdot N\left(I_{2}\right)_{H} \subseteq I_{1} I_{2}$. Thus we have $\left(I_{1}\right)_{H} N\left(I_{2}\right)_{H} \subseteq\left(I_{1} I_{2}\right)_{H}$ and the result follows since $\left(I_{1}\right)_{H} \triangleleft R * H$. 
(ii) Since $L_{2}{ }^{G} \triangleleft R * G$ we have $\bar{G} L_{2} G \subseteq L_{2} G$ and hence

$$
L_{1}{ }^{G} L_{2}{ }^{G} \subseteq L_{1} \bar{G} L_{2}{ }^{G} \subseteq L_{1} L_{2}{ }^{G} \subseteq L_{1} L_{2} \bar{G} .
$$

Thus since $L_{1}{ }^{G} L_{2} G \triangleleft R * G$, Lemma 1.5(ii) yields $L_{1}{ }^{G} L_{2} G \subseteq\left(L_{1} L_{2}\right)^{G}$.

LEMMA 1.7. (i) Let $L \triangleleft R * H$ with $L \cap R \supseteq Q$. Then $\bar{G} N L \bar{G} \subseteq L^{G} \subseteq L \bar{G}$ and $L \subseteq\left(L^{G}\right)_{H}$. Furthermore $N\left(L^{G}\right)_{H} \subseteq L$.

(ii) If $I \triangleleft R * G$, then $M\left(I_{H}\right)^{G} \subseteq I$. Moreover there exists a nonzero G-stable ideal $E$ of $R$ with $E I \subseteq\left(I_{H}\right)^{G}$.

Proof. (i) If $x \in H$, then $\bar{x} N L \bar{G} \subseteq L \bar{G}$ since $L$ is an ideal of $R * H$. If $x \notin H$ then

$$
\bar{x} N L \bar{G}=N^{\bar{x}^{-1}} L^{\bar{x}^{-1}} \bar{G} \subseteq Q(R * G) \subseteq L \bar{G}
$$

since $N^{\bar{x}^{-1}} \subseteq Q \subseteq L$ for $x \notin H$. Thus $\bar{G} N L \bar{G} \subseteq L \bar{G}$ and since $\bar{G} N L \bar{G} \triangleleft R * G$ we have $\bar{G} N L \bar{G} \subseteq L^{G} \subseteq L \bar{G}$. In particular $N L \subseteq L^{G}$ so $L \subseteq\left(L^{G}\right)_{H}$. In the other direction, $N\left(L^{G}\right)_{H} \subseteq L^{G} \subseteq L \bar{G}$ so clearly $N\left(L^{G}\right)_{H} \subseteq L$.

(ii) We have $N\left(I_{H}\right)^{G} \subseteq N I_{H} \bar{G} \subseteq I \bar{G}=I$, where the second inclusion holds by definition of $I_{H}$. Since $I$ and $\left(I_{H}\right)^{G}$ are both $G$-stable it then follows that $M\left(I_{H}\right)^{G} \subseteq I$. In the other direction, we know by Lemma 1.4(ii) that $E I \subseteq$ $\bar{G} N(I \cap R * H) \bar{G}$ for a suitable nonzero $G$-invariant ideal $E$ of $R$. Furthermore $I \cap R * H \subseteq I_{H}$ and $I_{H} \triangleleft R * H$ with $I_{H} \supseteq Q=\operatorname{ann}_{R} N$. Thus by (i) above

$$
E I \subseteq \bar{G} N(I \cap R * H) \bar{G} \subseteq \bar{G} N I_{H} \bar{G} \subseteq\left(I_{H}\right)^{G}
$$

as required.

The following is the main result of this section.

THEOREM 1.8. Let $R * G$ be given with $R$ a $G$-prime right Noetherian ring and with $G$ polycyclic-by-finite. Let $Q$ be a minimal prime of $R$ and let $H$ be its stabilizer in $G$ so that $|G: H|<\infty$. Then the maps $P \rightarrow P_{H}, L \rightarrow L^{G}$ as described above yield a one-to-one correspondence between the prime ideals $P$ of $R * G$ with $P \cap R=0$ and the primes $L$ of $R * H$ with $L \cap R=Q$.

ProOF. We start with an observation on a form of cancellation. Let $L$ be a prime ideal of $R * H$ with $L \cap R=Q$ and suppose $E I \subseteq L^{G}$ where $I \triangleleft R * G$ and $E$ is a nonzero $G$-stable ideal of $R$. Then $E I \subseteq L \bar{G}$ so by applying the trace map $\operatorname{tr}_{H}$ we have $E \operatorname{tr}_{H}(I) \subseteq L$ and hence $(E * H) \operatorname{tr}_{H}(I) \subseteq L$. But certainly $E * H \nsubseteq L$ since $E$ is $G$-stable, $L \cap R=Q$ and $\bigcap_{x} Q^{x}=0$. Thus since $L$ is prime we deduce that $\operatorname{tr}_{H}(I) \subseteq L$ and hence $I \subseteq L^{G}$ by Lemma 1.5 (iii).

Now let $P$ be a prime ideal of $R * G$ with $P \cap R=0$ and set $L=P_{H}$. By Lemma 1.5(i), $L \cap R=P_{H} \cap R=Q$. Let us first observe, by Lemma 1.7(ii) that $P \supseteq M\left(P_{H}\right)^{G}=(M * G)\left(P_{H}\right)^{G}$. Thus since $P$ is prime and $M * G \nsubseteq P$, we see that $P \supseteq\left(P_{H}\right)^{G}=L^{G}$. Next we show that $L$ is prime. Indeed if $L_{1}$ and $L_{2}$ are ideals of $R * H$ containing $L$ with $L \supseteq L_{1} L_{2}$, then Lemma 1.6(ii) yields

$$
P \supseteq L^{G} \supseteq\left(L_{1} L_{2}\right)^{G} \supseteq L_{1}^{G} L_{2}{ }^{G} \text {. }
$$

Hence since $P$ is prime, $P \supseteq L_{i}{ }^{G}$ for some $i$ and then, by Lemma 1.7(i), since $L_{i} \cap R \supseteq L \cap R=Q$, we have $L=P_{H} \supseteq\left(L_{i}{ }^{G}\right)_{H} \supseteq L_{i}$. Thus $L$ is a prime ideal of $R * H$ with $L \cap R=Q$. Finally by Lemma 1.7 (ii) there exists a nonzero $G$-stable ideal $E$ of $R$ with $E P \subseteq\left(P_{H}\right)^{G}=L^{G}$. Hence by the cancellation property of $L^{G}$ 
mentioned above we have $P \subseteq L^{G}$ so $P=L^{G}$ and this half of the correspondence is proved.

In the other direction let $L$ be a prime ideal of $R * H$ with $L \cap R=Q$ and set $P=$ $L^{G}$. By Lemma 1.5(ii) we have $P \cap R=0$ and, by Lemma 1.7(i), $L \subseteq\left(L^{G}\right)_{H}=P_{H}$ and $L \supseteq N\left(L^{G}\right)_{H}=(N * H) P_{H}$. But $L$ is a prime ideal of $R * H$ and $L \nsupseteq N * H$, since $N \cap Q=0$, so the latter yields $L \supseteq P_{H}$ and hence $L=P_{H}$. Next we show that $P$ is a prime ideal of $R * G$. To this end let $I_{1}, I_{2} \triangleleft R * G$ with $I_{1} I_{2} \subseteq P$. Then by Lemma 1.6(i)

$$
\left(I_{1}\right)_{H}(N * H)\left(I_{2}\right)_{H} \subseteq\left(I_{1} I_{2}\right)_{H} \subseteq P_{H}=L
$$

and thus since $L$ is prime and $L \nsupseteq N * H$ we have $L \supseteq\left(I_{i}\right)_{H}$ for some $i$. Now applying Lemma 1.7(ii) to $I_{i}$ we obtain $E I_{i} \subseteq\left(I_{i}\right)_{H}{ }^{G} \subseteq L^{G}$ for some nonzero $G$-stable ideal $E$ of $R$. Hence, by the cancellation property for $L^{G}$, we have $I_{i} \subseteq$ $L^{G}=P$ and $P$ is prime. Since $L=P_{H}$, the result follows.

Finally we observe, with the above notation, that there is an obvious one-to-one correspondence between the prime ideals $L$ of $R * H$ with $L \cap R=Q$ and the prime ideals $\tilde{L}$ of

$$
(R * H) /(Q * H)=(R / Q) * H=\tilde{R} * H
$$

with $\tilde{L} \cap \tilde{R}=0$. Since $\tilde{R}$ is prime, we have therefore reduced the study of prime ideals in $R * G$ with $R$ a $G$-prime ring to those of $\tilde{R} * H$ with $\tilde{R}$ a prime ring. We consider the latter situation in the next section.

We note also that the Noetherian assumption is used only twice in this section. First it yields the existence of the prime ideal $Q$ of $R$ having only finitely many $G$-conjugates and satisfying $\bigcap_{x} Q^{x}=0$. However if we merely assume that $Q$ exists, then most of the work here is valid in this more general context. The second use occurs at the end of the proof of Lemma 1.4(ii). Again most of that result, if properly formulated, holds more generally. In particular, the proof shows that if $X$ is any finite subset of $G$, then there exists a nonzero ideal $E$ of $R$ with $E \subseteq N$ which satisfies $E \cdot(I \cap R * X) \subseteq N \cdot(I \cap R * H) \bar{G}$.

2. Prime coefficient rings. In this section we study crossed products $R * G$ with $R$ prime and we describe its prime ideals $P$ with $P \cap R=0$. The goal is to show that these correspond in a natural manner to the $G$-prime ideals of a certain twisted group algebra. For the most part the argument goes through without any additional assumptions on $R$ and $G$. However at the last step we require a Noetherian hypothesis. The work here closely parallels that of $[\boldsymbol{7}]$ on enveloping algebras but it is surprisingly easier.

It will be necessary to localize the prime ring $R$. Specifically we extend $R$ to its symmetric Martindale ring of quotients $Q_{s}(R)$. The latter exists and is uniquely characterized by the following four properties:

1. $Q_{s}(R) \supseteq R$ with the same 1 .

2. If $q \in Q_{s}$ then there exist $0 \neq A, B \triangleleft R$ with $A q, q B \subseteq R$.

3. If $q \in Q_{s}$ and $0 \neq I \triangleleft R$ then either $I q=0$ or $q I=0$ implies that $q=0$.

4. Let $f:{ }_{R} A \rightarrow{ }_{R} R$ and $g: B_{R} \rightarrow R_{R}$ be given with $0 \neq A, B \triangleleft R$ and suppose that for all $a \in A, b \in B$ we have $(a f) b=a(g b)$. Then there exists $q \in Q_{s}$ with $a f=a q$ and $g b=q b$ for all $a \in A, b \in B$.

For details see $[6, \S 1]$. In the following lemma we list some basic properties of $Q_{s}(R)$. 
LEMMA 2.1. Let $R$ be a prime ring and set $S=Q_{s}(R)$.

(i) $\mathbf{Z}(S)=\mathbf{C}_{S}(R)$ is a field called the extended centroid of $R$.

(ii) Any automorphism of $R$ extends uniquely to one of $S$. $S * G$.

(iii) If $R * G$ is given then there exists a unique extension to a crossed product

(iv) Let $\sigma \in$ Aut $R$ and let $a, b, c, d$ be nonzero elements of $S$. Suppose that for all $r \in R$ we have arb $=c r^{\sigma} d$. Then there exists a unit $q \in S$ with $r^{\sigma}=r^{q}=q^{-1} r q$ for all $r \in R$ and with $a=c q^{-1}, b=q d$.

ProOF. These exist in the literature in various forms for the left Martindale ring of quotients so we will not repeat the arguments here. For example an obvious modification of the proof of $[\mathbf{4}$, Lemma $2.1(\mathrm{v})$, (iv)] yields (i) and (ii) and [4, Lemma 2.3] yields (iii). Finally (iv) is an extension of [4, Lemma 2.2] with essentially the same proof.

We will require the following two technical lemmas

LEMMA 2.2. Let $F$ be a field, let $S$ and $T$ be $F$-algebras and let $\Gamma=\left(S \otimes_{F} T\right) * H$ be a crossed product. Assume that $\bar{H}$ normalizes both $S$ and $T$ and let $I$ be an $\bar{H}$ stable ideal of $T$. Then

$$
\begin{aligned}
\Gamma / I \Gamma & =(S \otimes T) * H /(S \otimes I) * H \\
& \simeq\left(S^{\prime} \otimes T^{\prime}\right) * H=\Gamma^{\prime}
\end{aligned}
$$

where $S^{\prime} \simeq S$ and $T^{\prime}=T / I$. Furthermore $\mathbf{C}_{\Gamma^{\prime}}\left(S^{\prime}\right)=\mathbf{C}_{\Gamma}(S)^{\prime}$, where the latter is the image of $\mathbf{C}_{\Gamma}(S)$.

Proof. Since $I \triangleleft T$ is $\bar{H}$-stable, it is clear that $S \otimes I \triangleleft S \otimes T$ is $H$-stable. Thus $I \Gamma=(S \otimes I) * H \triangleleft \Gamma$ and it follows from Lemma 1.1(i) that

$$
\begin{aligned}
\Gamma / I \Gamma & =(S \otimes T) * H /(S \otimes I) * H \\
& \simeq(S \otimes T / S \otimes I) * H .
\end{aligned}
$$

But $(S \otimes T) /(S \otimes I) \simeq S \otimes(T / I)$ so we have obtained the appropriate structure for $\Gamma^{\prime}=\Gamma / I \Gamma$.

It remains to consider the centralizers and certainly $\mathbf{C}_{\Gamma}(S)^{\prime} \subseteq \mathbf{C}_{\Gamma^{\prime}}\left(S^{\prime}\right)$, that is the centralizer of $S$ in $\Gamma$ maps into the centralizer of $S^{\prime}$ in $\Gamma^{\prime}$. For the other direction, let $\left\{t_{1}, t_{2}, \ldots\right\}$ be an $F$-basis for a complement for $I$ in $T$ and let $\alpha^{\prime} \in \mathbf{C}_{\Gamma^{\prime}}\left(S^{\prime}\right)$. Then we may assume that $\alpha$, an inverse image of $\alpha^{\prime}$, is of the form

$$
\alpha=\sum_{i, x}\left(a_{i x} \otimes t_{i}\right) \bar{x}
$$

with $a_{i x} \in S$. Let $s \in S$. Since $\bar{H}$ normalizes $S$ we have

$$
s \alpha-\alpha s=\sum_{i, x}\left[\left(s a_{i x}-a_{i x} s^{\bar{x}^{-1}}\right) \otimes t_{i}\right] \bar{x} .
$$

On the other hand, $s \alpha-\alpha s \in(S \otimes I) * H$ since $\alpha^{\prime} \in \mathbf{C}_{\Gamma^{\prime}}\left(S^{\prime}\right)$. By definition of $\left\{t_{i}\right\}$ it therefore follows that $s \alpha-\alpha s=0$. Since this is true for all $s \in S$ we have $\alpha \in \mathbf{C}_{\Gamma}(S)$ and hence $\alpha^{\prime} \in \mathbf{C}_{\Gamma}(S)^{\prime}$. 
LEMMA 2.3. $\Gamma=\left(S \otimes_{F} T\right) * H$ be as in the previous lemma. In addition assume that $R$ is a prime ring, $S=Q_{s}(R)$ and that $\bar{H}$ normalizes $R, S$, and $T$. If $I$ is a nonzero $(R, R)$-subbimodule of $\Gamma$ with $I \bar{H}=I$ then there exists $0 \neq \alpha \in \mathbf{C}_{\Gamma}(S)$ and $0 \neq A \triangleleft R$ with $A \alpha \subseteq I$.

Proof. Let $\left\{t_{0}, t_{1}, \ldots\right\}$ be an $F$-basis for $T$. Then every element $\beta \in \Gamma$ is uniquely writable as

$$
\beta=\sum_{i, x}\left(b_{i x} \otimes t_{i}\right) \bar{x}
$$

with $b_{i x} \in S$. Choose $0 \neq \beta \in I$ with a minimal number, say $n$, of nonzero coefficients $b_{i x}$. We may suppose $b_{0 y} \neq 0$.

Let $r \in R$. Since $I$ is an $(R, R)$-subbimodule of $\Gamma$ we have

$$
\gamma=b_{0 y} r \beta-\beta r^{\bar{y}} b_{0 y}^{\bar{y}} \in I \text {. }
$$

Furthermore

$$
\gamma=\sum_{i, x}\left[\left(b_{0 y} r b_{i x}-b_{i x} r^{\bar{y} \bar{x}^{-1}} b_{0 y}^{\bar{y} \bar{x}^{-1}}\right) \otimes t_{i}\right] \bar{x}
$$

and the $(0, y)$-term here is zero. Thus the minimality of $n$ implies that $\gamma=0$ and hence that

$$
b_{0 y} r b_{i x}=b_{i x} r^{\bar{y} \bar{x}^{-1}} b_{0 y}^{\bar{y} \bar{x}^{-1}}
$$

for all $r \in R$. It follows from Lemma 2.1(iv) that there exist units $q_{i x} \in S$ with

$$
\bar{x} \bar{y}^{-1} r \bar{y} \bar{x}^{-1}=r^{\bar{y} \bar{x}^{-1}}=q_{i x}^{-1} r q_{i x}
$$

and $b_{i x}=b_{0 y} q_{i x}$ for all $i, x$ which occur in the support of $\beta$.

Set

$$
\alpha=\sum_{i, x}\left(q_{i x} \otimes t_{i}\right) \bar{x} \bar{y}^{-1} .
$$

Since $q_{i x} \bar{x} \bar{y}^{-1}$ centralizes $R$ and normalizes $S$, it follows from the uniqueness part of Lemma 2.1(ii) that $q_{i x} \bar{x} \bar{y}^{-1}$ centralizes $S$ and hence that $0 \neq \alpha \in \mathbf{C}_{\Gamma}(S)$. Furthermore since $b_{i x}=b_{0 y} q_{i x}$ we have

$$
b_{0 y} \alpha=\sum_{i, x}\left(b_{i x} \otimes t_{i}\right) \bar{x} \bar{y}^{-1}=\beta \bar{y}^{-1} \in I
$$

since $I \bar{H}=I$ implies $I \bar{H}^{-1}=I$.

Finally there exists an ideal $A$ of $R$ with $0 \neq A b_{0 y} \subseteq R$. Thus since $I$ is an $(R, R)$-bimodule and $\alpha$ commutes with $R$ we have

$$
\left(A b_{0 y} R\right) \alpha=A\left(b_{0 y} \alpha\right) R \subseteq I
$$

and the lemma is proved.

We now fix some notation for the remainder of this section. Let $\Gamma=R * G$ be a crossed product with $R$ a prime ring. By Lemma 2.1(iii), if $S=Q_{s}(R)$ then $\Gamma$ extends uniquely to a crossed product $\Gamma^{\prime}=S * G$. Set $F=\mathbf{Z}(S)=\mathbf{C}_{S}(R)$, the extended centroid of $R$. Recall that an automorphism $\sigma$ of $R$ is said to be $X$-inner if its unique extension to $S$ becomes inner. Since conjugation by every unit of $R$ is obviously $X$-inner it follows that

$$
G_{\text {inn }}=\left\{\left.x \in G\right|^{\bar{x}} \text { is } X \text {-inner on } R\right\}
$$

is a subgroup of $G$ and in fact $G_{\text {inn }} \triangleleft G$. 
LEMMA 2.4. If $T=\mathbf{C}_{\Gamma^{\prime}}(S)$ then $T=F^{t}\left[G_{\mathrm{inn}}\right]$, some twisted group algebra of $G_{\text {inn }}$ over $F$, and $G$ acts on $T$ normalizing the group of trivial units. Furthermore $S * G_{\mathrm{inn}}=S \otimes_{F} T$ and $\Gamma^{\prime}=\left(S * G_{\mathrm{inn}}\right) * H=\left(S \otimes_{F} T\right) * H$, a suitable crossed product of $H=G / G_{\text {inn }}$ over $S \otimes T$.

Proof. It is clear that $\alpha=\sum a_{x} \bar{x} \in T=\mathbf{C}_{\Gamma^{\prime}}(S)$ if and only if each $a_{x} \bar{x} \in T$. Now suppose $s \bar{x} \in T$ with $0 \neq s \in S$. Then for all $r \in R$ we have

$$
r s \bar{x}=s \bar{x} r=s r^{\bar{x}^{-1}} \bar{x}
$$

and thus, by Lemma 2.1(iv), $s$ is a unit of $S$ with $s^{-1} r s=r^{\bar{x}^{-1}}$ for all $r \in R$. Thus $x \in G_{\text {inn }}$ and it follows that $T \subseteq S * G_{\text {inn }}$.

For each $x \in G_{\text {inn }}$ choose a unit $s_{x} \in S$ inducing the automorphism ${ }^{\bar{x}}$ on $R$ and let $\tilde{x}=s_{x}^{-1} \bar{x}$. We claim that the elements $\tilde{x}$ for all $x \in G_{\text {inn }}$ form an $S$-basis for $S * G_{\text {inn }}$ and an $F$-basis for $T$. The former is obvious and for the latter we know at least that the $\tilde{x}$ 's are $F$-linearly independent. Observe that $\tilde{x}$ acts on $S$ by conjugation and centralizes $R$. Thus $\tilde{x}$ centralizes $S$ so $\tilde{x} \in T$. Finally suppose that $\beta \in T \subseteq S * G_{\text {inn }}$. Then we can write $\beta=\sum b_{x} \tilde{x}$ with $b_{x} \in S$. Since each $b_{x} \tilde{x} \in T$ and since $\tilde{x}$ is a unit in $T$, it follows that $b_{x} \in \mathbf{Z}(S)=F$. We conclude that the elements $\tilde{x}$ do indeed form an $F$-basis for $T$ and $S * G_{\text {inn }}=S \otimes_{F} T$.

Now $T$ is an $F$-algebra with basis $\tilde{G}_{\text {inn }}$ and for each $x, y \in G_{\text {inn }}$ we have $\tilde{x} \tilde{y}=s \widetilde{x y}$ for some $0 \neq s \in S$. Since $\tilde{x}, \tilde{y}, \widetilde{x y} \in T$ we conclude that $s \in T$ so $s \in F$ and $T=F^{t}\left[G_{\text {inn }}\right]$ is a suitable twisted group algebra of $G_{\text {inn }}$ over $F$. Note that $\mathfrak{G}$ normalizes $S$ so it normalizes $T$ and then clearly normalizes the group of trivial units of $T$. Furthermore $U=\mathcal{U}(R)$ centralizes $T$ so we obtain an action of $G \simeq \mathfrak{G} / U$ on $T$. The remaining property, namely that $\Gamma^{\prime}=\left(S * G_{\mathrm{inn}}\right) *\left(G / G_{\mathrm{inn}}\right)$, is clear since $G_{\text {inn }} \triangleleft G$.

For the remainder of this section we will write $T=\mathbf{C}_{\Gamma^{\prime}}(S)$. In addition, if $I$ is a $(\Gamma, \Gamma)$-subbimodule of $\Gamma^{\prime}=S * G$ we set

$$
\tilde{I}=\{\alpha \in T \mid A \alpha \subseteq I \text { for some } 0 \neq A \triangleleft R\} .
$$

LEMMA 2.5. With the above notation, $\tilde{I}$ is a G-stable ideal of $T$. Furthermore if $\beta \in \tilde{I} \Gamma^{\prime}$ then there exists $0 \neq B \triangleleft R$ with $B \beta \subseteq I$.

Proof. If $\alpha, \beta \in \tilde{I}$ with $A \alpha, B \beta \subseteq I$, then $(A \cap B)(\alpha+\beta) \subseteq I$ so $\alpha+\beta \in \tilde{I}$. Now let $\gamma \in T$ and choose $0 \neq C \triangleleft R$ with $C \gamma \subseteq \Gamma$. Since $\alpha$ and $\gamma$ centralize $R$ we have

$$
A C \alpha \gamma=(A \alpha)(C \gamma) \subseteq I \Gamma=I
$$

and

$$
C A \gamma \alpha=(C \gamma)(A \alpha) \subseteq \Gamma I=I
$$

so $\alpha \gamma, \gamma \alpha \in \tilde{I}$. Thus $\tilde{I} \triangleleft T$. Furthermore since $I$ and $R$ are $\bar{G}$-stable, so is $\tilde{I}$.

Finally if $\beta \in \tilde{I} \Gamma^{\prime}$ then $\beta=\sum_{1}^{n} \alpha_{i} \gamma_{i}$ with $\alpha_{i} \in \tilde{I}$ and $\gamma_{i} \in \Gamma^{\prime}$. Choose $0 \neq A, C \triangleleft R$ with $A \alpha_{i} \subseteq I$ and $C \gamma_{i} \subseteq \Gamma$ for all $i=1,2, \ldots, n$. Then

$$
A C \beta \subseteq \sum_{1}^{n} A C \alpha_{i} \gamma_{i}=\sum_{1}^{n}\left(A \alpha_{i}\right)\left(C \gamma_{i}\right) \subseteq I \Gamma=I
$$

and the lemma is proved.

The following result is crucial. It applies when $I \triangleleft \Gamma$ or $I \triangleleft \Gamma^{\prime}$. 
LEMMA 2.6. If $I$ is a ( $\Gamma, \Gamma)$-subbimodule of $\Gamma^{\prime}$, then $\tilde{I} \Gamma^{\prime} \triangleleft \Gamma^{\prime}$ and $I \subseteq \tilde{I} \Gamma^{\prime}$.

Proof. By Lemma 2.4, $\Gamma^{\prime}=\left(S \otimes_{F} T\right) * H$ where $H=G / G_{\text {inn }}$ and, choosing $\bar{H} \subseteq \bar{G}$, we see that $R, S$ and $T$ are $\bar{H}$-invariant. Furthermore $\tilde{I}$ is an $\bar{H}$-stable ideal of $T$. By Lemma 2.2, $\tilde{I} \Gamma^{\prime} \triangleleft \Gamma^{\prime}$ and

$$
\Gamma^{\prime} / \tilde{I} \Gamma^{\prime}=(S \otimes(T / \tilde{I})) * H=\Gamma^{\prime \prime} .
$$

Furthermore $T=\mathbf{C}_{\Gamma^{\prime}}(S)$ maps onto the centralizer of $S$ in this quotient.

Suppose $I \nsubseteq \tilde{I} \Gamma^{\prime}$. Then the image $I^{\prime \prime}$ of $I$ in $\Gamma^{\prime \prime}$ is a nonzero $(R, R)$-subbimodule of $\Gamma^{\prime \prime}$ with $I^{\prime \prime} \bar{H}=I^{\prime \prime}$. It follows from Lemma 2.3 that there exists $0 \neq \alpha^{\prime \prime} \in \mathbf{C}_{\Gamma^{\prime \prime}}(S)$ with $A \alpha^{\prime \prime} \subseteq I^{\prime \prime}$ for some $0 \neq A \subseteq R$. As we observed, $\alpha^{\prime \prime}$ is the image of some element $\alpha \in T$ and certainly $A \alpha \subseteq I+\tilde{I} \Gamma^{\prime}$. Choose $a \in A$ with $a \neq 0$ and write $a \alpha=\gamma+\beta$ with $\gamma \in I$ and $\beta \in \tilde{I} \Gamma^{\prime}$. Then, by Lemma 2.5 , there exists $0 \neq B \triangleleft R$ with $B \beta \subseteq I$. Since $B \gamma$ is certainly contained in $I$ we have $B a \alpha \subseteq I$ and hence, since $\alpha$ centralizes $R$, we obtain $(B a R) \alpha=B a \alpha R \subseteq I R=I$. But $0 \neq B a R \triangleleft R$ implies that $\alpha \in \tilde{I}$, by definition of $\tilde{I}$, and therefore the image $\alpha^{\prime \prime}$ of $\alpha$ is zero, a contradiction. We conclude that $I \subseteq \tilde{I} \Gamma^{\prime}$.

Recall that $G$ acts on $T$. If $Q \neq T$ is a $G$-stable ideal, then $Q$ is $G$-prime if for all $G$-stable ideals $I, J$ of $T$ the inclusion $I J \subseteq Q$ implies $I \subseteq Q$ or $J \subseteq Q$. We can now set up the correspondence between the primes of $\Gamma=R * G$ disjoint from $R$ and the $G$-prime ideals of $T$. With little additional work we can add to this link the primes of $\Gamma^{\prime}=S * G$ disjoint from $S$.

LEMMA 2.7. Let $Q$ be a $G$-stable ideal of $T$ and set $P=Q \Gamma^{\prime} \cap \Gamma, P^{\prime}=Q \Gamma^{\prime}$. Then $P \triangleleft \Gamma$ and $P^{\prime} \triangleleft \Gamma^{\prime}$ with $P \cap R=P^{\prime} \cap S=0$ and $\tilde{P}=\tilde{P}^{\prime}=Q$. Furthermore if $Q$ is $G$-prime then $P$ is a prime ideal of $\Gamma$ and $P^{\prime}$ is a prime ideal of $\Gamma^{\prime}$.

ProOF. We apply Lemma 2.4 and write $\Gamma^{\prime}=\left(S \otimes_{F} T\right) * H$ where $H=G / G_{\text {inn }}$. Since $Q$ is a $G$-stable ideal of $T$, it follows that $S \otimes Q$ is an ideal of $S \otimes T$ which is $H$-stable. Thus by Lemma 1.1 (i) we have

$$
P^{\prime}=Q \Gamma^{\prime}=(S \otimes Q) * H \triangleleft \Gamma^{\prime}
$$

and $P^{\prime} \cap(S \otimes T)=S \otimes Q$. Thus $P=P^{\prime} \cap \Gamma \triangleleft \Gamma$ and $P \cap(S \otimes T) \subseteq S \otimes Q$. This implies that $P^{\prime} \cap S=P \cap R=0$.

Let $\alpha \in \tilde{P}$ or $\tilde{P}^{\prime}$. Then by definition there exists $0 \neq A \triangleleft R$ with

$$
A \alpha \subseteq P^{\prime} \cap(S \otimes T)=S \otimes Q
$$

and hence $\alpha \in Q$. Conversely if $\beta \in Q$ then there exists $0 \neq B \triangleleft R$ with $B \beta \subseteq \Gamma$ and thus

$$
B \beta=\beta B \subseteq Q \Gamma^{\prime} \cap \Gamma=P \subseteq P^{\prime} .
$$

We conclude therefore that $\tilde{P}=\tilde{P}^{\prime}=Q$.

Finally assume that $Q$ is $G$-prime. We show that $P$ is prime, the proof for $P^{\prime}$ being identical. Let $I, J \triangleleft \Gamma$ with $I J \subseteq P$ and let $\alpha \in \tilde{I}, \beta \in \tilde{J}$. If $0 \neq A, B \triangleleft R$ with $A \alpha \subseteq I, B \beta \subseteq J$ then

$$
A B \alpha \beta=(A \alpha)(B \beta) \subseteq I J \subseteq P
$$

so $\alpha \beta \in \tilde{P}$. In other words, $\tilde{I} \tilde{J} \subseteq \tilde{P}=Q$. But $\tilde{I}$ and $\tilde{J}$ are $G$-stable ideals of $T$, by Lemma 2.5 , and $Q$ is $G$-prime. It follows that one of $\tilde{I}$ or $\tilde{J}$ is contained in $Q$, say 
$\tilde{I} \subseteq Q$. We conclude from Lemma 2.6 that

$$
I \subseteq \tilde{I} \Gamma^{\prime} \cap \Gamma \subseteq Q \Gamma^{\prime} \cap \Gamma=P
$$

and the lemma is proved.

For the last step we require a Noetherian hypothesis. In some sense it is used to compensate for the fact that the ideals $A$ of $R$ we have considered are not necessarily $G$-stable.

LEMMA 2.8. Assume in addition that $R$ is right Noetherian and $G$ is polycyclicby-finite. Let $P$ be a prime ideal of $R * G$ with $P \cap R=0$ or let $P^{\prime}$ be a prime ideal of $S * G$ with $P^{\prime} \cap S=0$. Then $\tilde{P}$ and $\tilde{P}^{\prime}$ are $G$-prime ideals of $T$ with $P=\tilde{P} \Gamma^{\prime} \cap \Gamma, P^{\prime}=\tilde{P}^{\prime} \Gamma^{\prime}$.

Proof. We first show that $P=\tilde{P} \Gamma^{\prime} \cap \Gamma$. To this end let $W=\tilde{P} \Gamma^{\prime} \cap \Gamma$ so that $W \triangleleft \Gamma$ and $W \supseteq P$ by Lemma 2.6. Since $\Gamma$ is right Noetherian by Lemma 1.2 , we can write $W=\sum_{1}^{n} \alpha_{i} \Gamma$ and, since $\alpha_{i} \in W \subseteq \tilde{P} \Gamma^{\prime}$, it follows from Lemma 2.5 that there exists $0 \neq A_{i} \triangleleft R$ with $A_{i} \alpha_{i} \subseteq P$. Letting $A=\bigcap_{1}^{n} A_{i}$ we have $0 \neq A \triangleleft R$ and $A W \subseteq P$. Now $P$ is a prime ideal of $\Gamma$ and $W \triangleleft \Gamma$ so this implies that either $A \subseteq P$ or $W \subseteq P$. But by assumption $P \cap R=0$ so the first possibility cannot hold and we conclude that $W=P$ as required.

The argument for $P^{\prime}$ is slightly different since we are not given that $S$ is right Noetherian. But note that $T=F^{t}\left[G_{\text {inn }}\right]$. and $G_{\text {inn }}$ is polycyclic-by-finite so $T$ is right Noetherian by Lemma 1.2. Set $W^{\prime}=\tilde{P}^{\prime} \Gamma^{\prime}$ so that $W^{\prime} \triangleleft \Gamma^{\prime}$ and $W^{\prime} \supseteq P^{\prime}$. Since $T$ is right Noetherian, $\tilde{P}^{\prime}$ is a finitely generated right ideal of $T$ and hence $W^{\prime}=\tilde{P}^{\prime} \Gamma^{\prime}$ is a finitely generated right ideal of $\Gamma^{\prime}$. Now proceed as above.

Finally we show that $\tilde{P}$ and $\tilde{P}^{\prime}$ are $G$-prime. Since the proofs are identical in the two cases, we will only consider $\tilde{P}$. Let $I$ and $J$ be $G$-stable ideals of $T$ with $I J \subseteq \tilde{P}$. Since $I \Gamma^{\prime}$ and $J \Gamma^{\prime}$ are ideals of $\Gamma^{\prime}$, by Lemma 2.7 , we have $\Gamma^{\prime} J \subseteq J \Gamma^{\prime}$ and hence $\left(I \Gamma^{\prime}\right)\left(J \Gamma^{\prime}\right) \subseteq I J \Gamma^{\prime} \subseteq \tilde{P} \Gamma^{\prime}$. It follows that

$$
\left(I \Gamma^{\prime} \cap \Gamma\right)\left(J \Gamma^{\prime} \cap \Gamma\right) \subseteq \tilde{P} \Gamma^{\prime} \cap \Gamma=P
$$

and thus since $P$ is prime one of these factors is in $P$, say $I \Gamma^{\prime} \cap \Gamma \subseteq P$. By Lemma 2.7 again this yields $I=\left(I \Gamma^{\prime} \cap \Gamma\right)^{\sim} \subseteq \tilde{P}$ so $\tilde{P}$ is $G$-prime. Similarly $\tilde{P}^{\prime}$ is $G$-prime.

The above two results now combine to form the main theorem of this section. At this point it is essentially all notation and requires no additional proof.

THEOREM 2.9. Let $\Gamma=R * G$ be a crossed product with $R$ prime and right Noetherian and with $G$ polycyclic-by-finite. Let $S=Q_{s}(R), F=\mathbf{Z}(S)$ and let $\Gamma^{\prime}=S * G$ be the natural extension. Then $T=\mathbf{C}_{\Gamma^{\prime}}(S)=F^{t}\left[G_{\mathrm{inn}}\right]$ is a twisted group algebra of the group $G_{\mathrm{inn}} \triangleleft G$ and $G$ acts on $T$ as automorphisms normalizing the group of trivial units. Furthermore there exist one-to-one order preserving correspondences between the primes $P$ of $\Gamma$ with $P \cap R=0$, the primes $P^{\prime}$ of $\Gamma^{\prime}$ with $P^{\prime} \cap S=0$, and the G-prime ideals $Q$ of $T$. Specifically these maps are given by

$$
Q \rightarrow Q \Gamma^{\prime}=P^{\prime}, \quad P^{\prime} \rightarrow P^{\prime} \cap \Gamma=P, \quad P \rightarrow \tilde{P}=Q
$$

where

$$
\tilde{P}=\{\alpha \in T \mid \text { there exists } 0 \neq A \triangleleft R \text { with } A \alpha \subseteq P\} .
$$

To proceed further we will have to describe the $G$-prime ideals in a twisted group algebra. We handle the prime ideals in the next section. 
3. Twisted group algebras. It remains to study the prime ideals in twisted group algebras of polycyclic-by-finite groups. Let $F^{t}[G]$ be such an algebra and let $\mathfrak{G}$, as usual, denote its group of trivial units. Then $\mathfrak{G} / F^{*} \simeq G$ and since $G$ is finitely generated there exists a finitely generated subgroup $X$ of $\mathfrak{G}$ with $X /\left(F^{\cdot} \cap X\right) \simeq G$. Furthermore $G$ is finitely presented, by [5, Lemma $12.3 .12($ iv)], so it follows from [5, Lemma 12.3.12(ii)] that $F^{*} \cap X$ is finitely generated as a normal subgroup of $X$. But $F^{*} \cap X$ is central in $X$ so we conclude that it is a finitely generated abelian group and hence that $X$ is polycyclic-by-finite.

Now the homomorphism $X \rightarrow \mathfrak{G} \subseteq F^{t}[G]$ extends to a ring homomorphism $F[X] \rightarrow F^{t}[G]$ which is clearly onto. Hence any prime ideal of $F^{t}[G]$ determines a prime ideal of $F[X]$. Since the prime ideals of $F[X]$ have all been described in [8 and 3], we can therefore transfer the appropriate information to $F^{t}[G]$. Our goal is to obtain a reasonable characterization of the primes of $F^{t}[G]$ in a fairly economical manner. The route we take is independent of the choice of $X$ and yields the necessary uniqueness properties almost immediately. There is presumably a good deal more which could be said here.

We follow the notation of [8]. If $G$ permutes a set $\Omega$, an element $\alpha \in \Omega$ is said to be orbital, or more precisely $G$-orbital, if the $G$-orbit of $\alpha$ is finite. In particular $G$ permutes its elements by conjugation and the set of orbital elements is

$$
\Delta(G)=\left\{x \in G|| G: \mathbf{C}_{G}(x) \mid<\infty\right\},
$$

the f.c. center of $G$. Furthermore $G$ permutes its subgroups by conjugation and hence $N \subseteq G$ is orbital if and only if $\left|G: \mathbf{N}_{G}(N)\right|<\infty$. A polycyclic-by-finite group $G$ is said to be orbitally sound if for every orbital subgroup $N$ of $G$ there exists $M \triangleleft G$ with $M \subseteq N$ and $|N: M|<\infty$.

LEMMA 3.1. Let $G$ be polycyclic-by-finite, let $Z$ be a central subgroup of $G$ and assume that $G / Z$ is orbitally sound. Define $W \triangleleft G$ to be minimal with $G \supseteq$ $W \supseteq Z$ and $G / W$ an elementary abelian 2-group. Then $|G: W|<\infty$ and any automorphism of $G$ which normalizes $Z$ also normalizes $W$.

(i) Let $N \subseteq W$ with $N$ orbital in $W$. If $N$ contains no nonidentity normal subgroup of $G$, then $|N: \mathbf{Z}(W) \cap N|<\infty$.

(ii) $W$ is orbitally sound.

ProOF. Since $G$ is finitely generated, its homomorphic images which are elementary abelian 2-groups are of bounded order. It follows that there exists a unique minimal $W$ with $G \supseteq W \supseteq Z$ and $G / W$ an elementary abelian 2-group. Thus $|G: W|<\infty$ and the uniqueness of $W$ yields the result on automorphisms.

(i) We proceed by induction on the Hirsch number $h(N)$. The case $h(N)=0$ is trivial so assume $h(N) \geq 1$. Since $|G: W|<\infty, N$ is orbital in $G$ so there exists $H \triangleleft G$ with $|G: H|<\infty$ such that $H$ normalizes $N$. We may assume that $W \supseteq H \supseteq Z$.

Since $G / Z$ is orbitally sound and $Z N / Z$ is an orbital subgroup, there exists $C \triangleleft G$ with $Z N \supseteq C \supseteq Z$ and $|Z N: C|<\infty$. Replacing $C$ by $C \cap H$, we may assume that $C \subseteq H$. We have $C=Z(N \cap C)$ so $|N: N \cap C|<\infty$. Note that $N \cap C$ is also orbital in $W$ and normalized by $H$, so replacing $N$ by $N \cap C$ we may assume that $C=Z N$. 
Since $N$ contains no nontrival normal subgroup of $G$ we have $Z \cap N=1$. Also $H \triangleleft G$ and $N \triangleleft H$, since $N \subseteq C \subseteq H$, so we have $[C, H] \triangleleft G$ and

$$
[C, H]=[Z N, H]=[N, H] \subseteq N .
$$

Thus $[C, H]=[N, H]=1$ so $N$ is central in $H$ and in particular $N$ is abelian. If $h(N) \geq 2$ we can write $N=N_{1} \times N_{2}$ with $h\left(N_{1}\right), h\left(N_{2}\right)<h(N)$. Since $N_{1}, N_{2}$ are central in $H$, they are orbital in $W$. It follows by induction that $\left|N_{1}: \mathbf{Z}(W) \cap N_{1}\right|<$ $\infty$ and $\left|N_{2}: \mathbf{Z}(W) \cap N_{2}\right|<\infty$ so $|N: \mathbf{Z}(W) \cap N|<\infty$, as required.

It remains to consider $h(N)=1$. Now there exists an integer $n$ with $Z^{n}$ and $N^{n}$ both torsion free abelian. Then $C^{n}=Z^{n} \times N^{n} \triangleleft G$ so replacing $N$ by $N^{n}$ we can assume that $D=T \times N \triangleleft G$ with $T=Z^{n}$ a torsion free central subgroup and $N$ infinite cyclic. Let us now think of $D$ as being additive so that the finite group $G / H$ acts linearly on $D$. Furthermore if $\mathcal{Q}$ denotes the field of rational numbers, we can let $G / H$ act on $D \otimes \mathcal{Q}$. Since $G / H$ acts in a completely reducible manner and $T \otimes \mathcal{Q}$ is a central subspace of codimension 1 we see that $G / H$ can be diagonalized. In fact each element of $G / H$ acts $\operatorname{like} \operatorname{diag}(1,1, \ldots, 1, \lambda)$ where $\lambda: G / H \rightarrow \mathcal{Q}^{\circ}$ is a linear character. This implies that $\lambda^{2}=1$ so, by definition of $W, W \subseteq \operatorname{ker} \lambda$ and hence $W$ centralizes $D \otimes \mathcal{Q}$. Thus $W$ centralizes $N \subseteq D \otimes \mathcal{Q}$ and (i) is proved.

(ii) Let $N \subseteq W$ with $N$ orbital in $W$. Set $M=\bigcap_{g} N^{g} \triangleleft G$ and consider $\bar{G}=$ $G / M$. We have $\bar{G} \supseteq \bar{Z}=Z M / M$ so $\bar{Z}$ is central in $\bar{G}$. Also $\bar{G} / \bar{Z} \simeq G / Z M$ is a homomorphic image of $G / Z$ so $\bar{G} / \bar{Z}$ is orbitally sound. Since $W \supseteq Z M$, it is clear that $\bar{W}$ is minimal in $\bar{G}$ subject to $\bar{W} \supseteq \bar{Z}$ and $\bar{G} / \bar{W}$ being an elementary abelian 2-group. Notice that $\bigcap_{\bar{g}} \bar{N}^{\bar{g}}=\bar{M}=1$ so (i) applies and we conclude that $|\bar{N}: \mathbf{Z}(\bar{W}) \cap \bar{N}|<\infty$. If $T$ denotes the complete inverse image of $\mathbf{Z}(\bar{W}) \cap \bar{N}$ in $G$, then clearly $T \triangleleft W$ and $|N: T|<\infty$. Thus $W$ is orbitally sound.

We remark that $G$ above need not be orbitally sound. For example let $G=$ $C$ wr $C_{2}$ be the wreath product of the infinite cyclic group $C$ by $C_{2}$, the group of order 2. If $Z=\mathbf{Z}(G)$, then $G / Z$ has Hirsch number 1 and hence is orbitally sound. But $G$ itself is not orbitally sound.

Let $G$ be polycyclic-by-finite. By [8, Theorem C2], $G_{0}=$ nio $G$ is a characteristic orbitally sound subgroup of $G$ of finite index. Let $G_{1}=\operatorname{nio}^{2} G$ be the subgroup of $G$ generated by the squares of all elements of nio $G$. Then $G_{0} / G_{1}$ is an elementary abelian 2-group and clearly the largest such homomorphic image of $G_{0}$.

PROPOSITION 3.2. If $G$ is polycyclic-by-finite, then nio $^{2} G$ is a characteristic subgroup of finite index. Furthermore let $X$ be any polycyclic-by-finite group such that $X / Z \simeq G$ for some central subgroup $Z$ of $X$. If $Y$ is the complete inverse image of nio $^{2} G$ in $X$, then $Y$ is orbitally sound.

Proof. Write $G_{0}=\operatorname{nio} G$ and $G_{1}=\operatorname{nio}^{2} G$. Then $\left|G: G_{0}\right|<\infty$ and $\left|G_{0}: G_{1}\right|<$ $\infty$ so $G_{1}$ is clearly a characteristic subgroup of $G$ of finite index. Now let $X / Z \simeq G$ and let $X_{i}$ be the complete inverse image of $G_{i}$. Then $X_{0} / Z \simeq G_{0}$ is orbitally sound so, by Lemma 3.1 and the definition of $G_{1}, X_{1}$ is orbitally sound.

If $G$ is polycyclic-by-finite and $N$ is orbital in $G$, then $N$ is an isolated orbital if any orbital $N_{1}$ properly larger than $N$ satisfies $\left|N_{1}: N\right|=\infty$. It turns out [8, $\S 3.1]$ that any orbital $N$ is a subgroup of finite index in a unique isolated orbital subgroup $i_{G}(N)$, its isolator. The isolated orbitals are important because their 
normalizers are largest among all nearby orbital subgroups. This latter concept is more useful in dealing with twisted group algebras.

If $G$ is any group and $N$ is a subgroup of $G$, we say that $N$ is bounding in $G$ if, for all subgroups $T$ of finite index in $N$, we have $\mathbf{N}_{G}(N) \supseteq \mathbf{N}_{G}(T)$. The next two lemmas list a number of properties of such subgroups. We note that if $H$ is a finitely generated group and $n$ is an integer, then $H$ has only finitely many subgroups of index $\leq n$ by [5, Lemma 6.3.3]. The intersection $T_{n}$ of these subgroups is therefore a characteristic subgroup of $H$ of finite index which is contained in any subgroup of index $\leq n$.

LEMMA 3.3. Let $G$ be a polycyclic-by-finite group and let $N$ be an orbital subgroup.

(i) If $N$ is $G$-bounding and $H \supseteq N$ with $|H: N|<\infty$, then $\mathbf{N}_{G}(N) \supseteq \mathbf{N}_{G}(H)$.

(ii) $N$ is G-bounding if and only if $\mathbf{N}_{G}(N)=\mathbf{N}_{G}\left(i_{G}(N)\right)$.

(iii) $N$ has a unique subgroup $b_{G}(N)$ which is maximal with the property that $\left|N: b_{G}(N)\right|<\infty$ and $b_{G}(N)$ is bounding in $G$. Indeed $b_{G}(N)$ is the largest normal subgroup of $\mathbf{N}_{G}\left(i_{G}(N)\right)$ contained in $N$.

Proof. (i) Choose $T$ char $H$ with $T \subseteq N \subseteq H$ and $|H: T|<\infty$. Since $T$ char $H$ we have $\mathbf{N}_{G}(H) \subseteq \mathbf{N}_{G}(T)$ and since $N$ is bounding we have $\mathbf{N}_{G}(T) \subseteq \mathbf{N}_{G}(N)$.

(ii) Let $I=i_{G}(N)$ and assume first that $N$ is bounding. Since $|I: N|<\infty$, (i) implies that $\mathbf{N}_{G}(N) \supseteq \mathbf{N}_{G}(I)$. On the other hand, since $I=i_{G}(N)$ we have $\mathbf{N}_{G}(N) \subseteq \mathbf{N}_{G}(I)$. Conversely assume that $\mathbf{N}_{G}(N)=\mathbf{N}_{G}(I)$ and let $T$ be a subgroup of $N$ of finite index. Then $T$ is orbital in $G$ and $i_{G}(T)=I$ so $\mathbf{N}_{G}(T) \subseteq$ $\mathbf{N}_{G}(I)=\mathbf{N}_{G}(N)$ and $N$ is bounding.

(iii) Again let $I=i_{G}(N)$ and set $M=\mathbf{N}_{G}(I)$. Since the finitely many $M$ conjugates of $N$ all have finite index in $I, B=\bigcap_{x \in M} N^{x} \triangleleft M$ also has finite index in I. Clearly $B$ is orbital, $i_{G}(B)=I$ and $B \triangleleft M$ so we have $\mathbf{N}_{G}(B)=\mathbf{N}_{G}\left(i_{G}(B)\right)=M$ and $B$ is bounding by (ii). Conversely, if $T \subseteq N$ is a $G$-bounding subgroup of finite index, then $T$ is orbital, $i_{G}(T)=i_{G}(N)=I$ and, by (ii), $T \triangleleft M$. Thus $T \subseteq \bigcap_{x \in M} N^{x}=B$ and $B$ has the appropriate property.

LEMMA 3.4. Let $G / \mathbf{Z}(G)$ be polycyclic-by-finite and let $N$ be a finitely generated orbital subgroup of $G$.

(i) $N$ has a unique subgroup $b_{G}(N)$ which is maximal subject to $\left|N: b_{G}(N)\right|<\infty$ and $b_{G}(N)$ is $G$-bounding.

(ii) If $X$ is any subgroup of $G$ with $G=X \mathbf{Z}(G)$ and $X \supseteq N$, then $b_{X}(N)=$ $b_{G}(N)$.

Proof. Let $X$ be any subgroup of $G$ with $G=X \mathbf{Z}(G)$ and $X \supseteq N$. If $T$ is any subgroup of $N$, then $\mathbf{N}_{G}(T)=\mathbf{N}_{X}(T) \mathbf{Z}(G)$ and $\mathbf{N}_{X}(T)=X \cap \mathbf{N}_{G}(T)$. It follows that $\mathbf{N}_{G}\left(T_{1}\right) \supseteq \mathbf{N}_{G}\left(T_{2}\right)$ if and only if $\mathbf{N}_{X}\left(T_{1}\right) \supseteq \mathbf{N}_{X}\left(T_{2}\right)$. In particular $T$ is $G$-bounding if and only if it is $X$-bounding.

Since $G / \mathbf{Z}(G)$ and $N$ are both finitely generated, we can now suppose that $X$ is finitely generated. As we observed at the beginning of this section this implies that $X$ is polycyclic-by-finite. By Lemma $3.3($ iii) $), b_{X}(N)$ exists and we conclude from the above that $b_{G}(N)$ exists and is equal to $b_{X}(N)$.

The prime ideals in group algebras of polycyclic-by-finite groups are characterized in [3 and 8]. The next result is merely a reformulation of the work of $[\mathbf{3}]$. 
Recall that if $I \triangleleft F[G]$ then

$$
I^{\dagger}=\{x \in G \mid 1-x \in I\}
$$

is a normal subgroup of $G$. Furthermore if $N \subseteq G$, then $\nabla_{G}(N) \supseteq N$ is defined by $\nabla_{G}(N) / N=\Delta\left(\mathbf{N}_{G}(N) / N\right)$. Note that if $N$ is a bounding orbital subgroup of $G$ and if $M=i_{G}(N)$, then $\mathbf{N}_{G}(N)=\mathbf{N}_{G}(M)$ by Lemma 3.3(ii). Since $|M: N|<\infty$ it then follows easily that $\nabla_{G}(N)=\nabla_{G}(M)$. We will use this observation freely below.

Since group algebras and twisted group algebras are crossed products, we can use the induced ideal notation $L^{G}$ as described immediately preceding Lemma 1.5 in either of these rings.

Proposition 3.5. Let $G$ be a polycyclic-by-finite group and let $H$ be a normal orbitally sound subgroup of finite index.

(i) Let $P$ be a prime ideal of $F[G]$ and write $P \cap F[H]=\bigcap_{x \in G} Q^{x}$ where $Q$ is a minimal covering prime of the intersection. If $N=b_{G}\left(Q^{\dagger}\right)$, then there exists a prime ideal $L$ of $F\left[\nabla_{G}(N)\right]$, unique up to conjugation by $\mathbf{N}_{G}(N)$, with $P=L^{G}$. Furthermore $L$ is $\mathbf{N}_{G}(N)$-orbital and $b_{G}\left(L^{\dagger}\right) \cap H=N$.

(ii) Conversely let $N$ be a bounding orbital subgroup of $G$ contained in $H$ and let $L$ be a prime ideal of $F\left[\nabla_{G}(N)\right]$ with $b_{G}\left(L^{\dagger}\right) \cap H=N$. Then $L^{G}=P$ is a prime ideal of $F[G]$ and there exists a minimal covering prime $Q$ of $P \cap F[H]$ with $N=b_{G}\left(Q^{\dagger}\right)$.

Proof. (i) Since $Q^{\dagger} \triangleleft H, Q^{\dagger}$ is certainly orbital in $G$. If $M=i_{G}\left(Q^{\dagger}\right)$ and $N=b_{G}\left(Q^{\dagger}\right)$, then $M=i_{G}(N)$ and $\nabla_{G}(M)=\nabla_{G}(N)$. With this observation [3, Theorem 2.3(iii)] yields all of (i) except for the uniqueness of $L$ and the information on $b_{G}\left(L^{\dagger}\right)$. For the remainder let $A=\mathbf{N}_{G}(M)=\mathbf{N}_{G}(N)$ and let $T=L^{A}$. Then by $[\mathbf{3}$, Theorem $2.3(\mathrm{ii})], T$ is a uniquely determined prime ideal of $F[A]$. We study $T^{\dagger}$.

Since $\nabla_{G}(M) \triangleleft A$ and $T=L^{A}$ we have $T=\left(\bigcap_{a \in A} L^{a}\right) F[A]$. It follows first that $T^{\dagger} \subseteq \nabla_{G}(M)$ and then that

$$
T^{\dagger}=\bigcap_{a \in A}\left(L^{\dagger}\right)^{a} .
$$

Next by $\left[\mathbf{3}\right.$, Theorem 2.3(ii)] again, $T \cap F[H]=\bigcap_{a \in A} Q^{a}$, so clearly

$$
T^{\dagger} \cap H=\bigcap_{a \in A}\left(Q^{\dagger}\right)^{a}=b_{G}\left(Q^{\dagger}\right)
$$

where the last equality follows from Lemma 3.3(iii). In particular $L^{\dagger} \supseteq T^{\dagger} \supseteq$ $b_{G}\left(Q^{\dagger}\right)=N$ and we see that $L$ is the complete inverse image of a prime ideal of $F\left[\nabla_{G}(M) / N\right]=F\left[\nabla_{G}(N) / N\right]$. But $A=\mathbf{N}_{G}(N)$ acts like a finite group of automorphisms on $\nabla_{G}(N) / N=\Delta(A / N)$ and therefore $L$ has only finitely many $A$-conjugates.

We conclude from this and $T \cap F\left[\nabla_{G}(N)\right]=\bigcap_{a \in A} L^{a}$ that $\left\{L^{a} \mid a \in A\right\}$ is the finite set of all minimal covering primes of the intersection. But $T$ is uniquely determined by $P$ and hence so is $\left\{L^{a}\right\}$ so we have proved the uniqueness of $L$ up to $A$-conjugation. In addition $L^{\dagger}$ is orbital in $A$ and hence in $G$.

Finally by $\left[3\right.$, Theorem 2.3(iii)] there exists at least one such prime $L_{1}$ with $\left|M: L_{1}^{\dagger}\right|<\infty$ and thus, by uniqueness, $\left|M: L^{\dagger}\right|<\infty$ for all such $L$. It follows that 
$M=i_{G}\left(L^{\dagger}\right)$ and hence that

$$
T^{\dagger}=\bigcap_{a \in A}\left(L^{\dagger}\right)^{a}=b_{G}\left(L^{\dagger}\right)
$$

by Lemma 3.3(iii). Since $T^{\dagger} \cap H=b_{G}\left(Q^{\dagger}\right)=N$, part (i) is proved.

(ii) Here we use [3, Theorem 2.4] which applies equally well to any orbitally sound normal subgroup $H$ of $G$ of finite index. Again if $M=i_{G}(N)$, then $A=$ $\mathbf{N}_{G}(M)=\mathbf{N}_{G}(N)$ and $\nabla_{G}(M)=\nabla_{G}(N)$. Now by assumption, $\nabla_{G}(N) \supseteq L^{\dagger} \supseteq N$ so $L^{\dagger}$ is orbital in $A$ and hence in $G$. Thus $b_{G}\left(L^{\dagger}\right)$ does indeed make sense and $b_{G}\left(L^{\dagger}\right) \cap H=N$ implies that $\left|L^{\dagger}: N\right|<\infty$ and $M=i_{G}\left(L^{\dagger}\right)$. By $[3$, Theorem 2.4 (i)], $P$ is a prime ideal of $F[G]$ and there exists a minimal covering prime $Q_{1}$ of $P \cap F[H]$ with $M=i_{G}\left(Q_{1}^{\dagger}\right)$. It remains to compute $b_{G}\left(Q_{1}^{\dagger}\right)$.

To this end let $N_{1}=b_{G}\left(Q_{1}^{\dagger}\right)$ and let $L_{1}$ be the prime ideal of $F\left[\nabla_{G}\left(N_{1}\right)\right]$ given by (i) with $P=L_{1}^{G}$. Since $M=i_{G}\left(N_{1}\right)$, it follows that $\nabla_{G}\left(N_{1}\right)=\nabla_{G}(M)=\nabla_{G}(N)$ so $L_{1}$ and $L$ are both prime ideals of $F\left[\nabla_{G}\left(N_{1}\right)\right]$ with $L^{G}=L_{1}^{G}=P$. By the uniqueness in (i) we therefore have $L_{1}^{a}=L$ for some $a \in A$. Then $\left(L_{1}^{\dagger}\right)^{a}=L^{\dagger}$ so $b_{G}\left(L_{1}^{\dagger}\right)^{a}=b_{G}\left(L^{\dagger}\right)$ and hence since $H \triangleleft G$,

$$
N_{1}^{a}=\left(b_{G}\left(L_{1}^{\dagger}\right) \cap H\right)^{a}=b_{G}\left(L_{1}^{\dagger}\right)^{a} \cap H=b_{G}\left(L^{\dagger}\right) \cap H=N .
$$

Since $N=N_{1}^{a}=b_{G}\left(Q_{1}^{\dagger}\right)^{a}$, the result follows with $Q=Q_{1}^{a}$.

We now require analogous notation for twisted group algebras. If $I \triangleleft F^{t}[G]$ we let

$$
I^{t}=\{\alpha \in \mathfrak{G} \mid 1-\alpha \in I\} .
$$

Then $I^{t}$ is the kernel of the group homomorphism $\mathfrak{G} \rightarrow F^{t}[G] / I$ so $I^{t} \triangleleft \mathfrak{G}$. Furthermore if $I \neq F^{t}[G]$, then $I^{t} \cap F^{*}=1$.

Again assume that $F^{t}[G]$ is given. If $N$ is a subgroup of $\mathfrak{G}$ we define $\nabla_{G}^{t}(N) \subseteq G$ as follows. First we form $\mathbf{N}_{\mathfrak{G}}(N)$ and then let $D \supseteq N$ be the complete inverse image in $\mathbf{N}_{\mathfrak{B}}(N)$ of the f.c. center $\Delta\left(\mathbf{N}_{\mathfrak{B}}(N) / N\right)$ so that $D=\nabla_{\mathfrak{B}}(N)$. Note that $\mathbf{N}_{\mathfrak{G}}(N) \supseteq D \supseteq F^{*}$ and we set

$$
\nabla_{G}^{t}(N)=D / F^{\cdot} \subseteq \mathfrak{G} / F^{\cdot}=G .
$$

We remark that if $\mathbf{N}_{\mathfrak{G}}(N) \supseteq M \supseteq N$ with $|M: N|<\infty$ and $\mathbf{N}_{\mathfrak{B}}(M)=\mathbf{N}_{\mathfrak{G}}(N)$, then it follows immediately that $\nabla_{G}^{t}(M)=\nabla_{G}^{t}(N)$. The main result of this section is

THEOREM 3.6. Let $F^{t}[G]$ be a twisted group algebra of the polycyclic-by-finite group $G$. Set $H=\operatorname{nio}^{2} G$ and let $\mathfrak{H}$ denote the group of trivial units of $F^{t}[H] \subseteq$ $F^{t}[G]$.

(i) Let $P$ be a prime ideal of $F^{t}[G]$ and write $P \cap F^{t}[H]=\bigcap_{x \in G} Q^{x}$ where $Q$ is a minimal covering prime of the intersection. If $N=b_{\mathfrak{G}}\left(Q^{t}\right)$ then there exists a prime ideal $L$ of $F^{t}\left[\Delta_{G}^{t}(N)\right]$, unique up to conjugation by $\mathbf{N}_{\mathfrak{B}}(N)$, with $P=L^{G}$. Furthermore $L$ is $\mathbf{N}_{\mathfrak{G}}(N)$-orbital and $b_{\mathfrak{G}}\left(L^{t}\right) \cap \mathfrak{H}=N$.

(ii) Conversely let $N$ be a bounding, orbital subgroup of $\mathfrak{G}$ contained in $\mathfrak{H}$ with $N \cap F^{*}=1$ and let $L$ be a prime ideal of $F^{t}\left[\nabla_{G}^{t}(N)\right]$ with $b_{\mathfrak{B}}\left(L^{t}\right) \cap \mathfrak{H}=N$. Then $P=L^{G}$ is a prime ideal of $F^{t}[G]$ and there exists a minimal covering prime $Q$ of $P \cap F^{t}[H]$ with $N=b_{\mathfrak{G}}\left(Q^{t}\right)$.

Proof. (i) Since $H=\operatorname{nio}^{2} G$ is a normal subgroup of $G$ of finite index, the structure of $P \cap F^{t}[H]$ is immediate from Lemma 1.3. Furthermore $Q^{t} \triangleleft \mathfrak{H}$ and 
$|\mathfrak{G}: \mathfrak{H}|<\infty$ so $Q^{t}$ is orbital in $\mathfrak{G}$ with $Q^{t} \cap F^{\bullet}=1$. The latter implies that $Q^{t}$ embeds isomorphically in $\mathfrak{G} / F^{*}=G$ so $Q^{t}$ is finitely generated. By Lemma 3.4(i), $b_{\mathfrak{G}}\left(Q^{t}\right)=N$ exists.

If there exists a prime ideal $L_{1}$ of $F^{t}\left[\nabla_{G}^{t}(N)\right]$ with $L_{1}^{G}=P$, then choose one such. Again $L_{1}^{t} \cap F^{*}=1$ so $L_{1}^{t}$ is finitely generated. We can now let $X$ be a finitely generated subgroup of $\mathfrak{G}$ with $F^{*} X=\mathfrak{G}$ and $X \supseteq Q^{t}, L_{1}^{t}$. As we observed at the beginning of this section, this implies that $X$ is polycyclic-by-finite. To avoid confusion we set $Z=X \cap F^{*}$, the kernel of the homomorphism of $X$ onto $G$.

Now the map $X \rightarrow \mathfrak{G} \subseteq F^{t}[G]$ extends to a ring epimorphism $F[X] \rightarrow F^{t}[G]$ and we pull certain information back to $F[X]$. In particular we let $\tilde{P}$ be the complete inverse image of $P$ in $F[X]$ and we let $X_{1}$ be the inverse image of $H=\operatorname{nio}^{2} G$ in $X$ so that $F\left[X_{1}\right]$ maps onto $F^{t}[H]$. In addition we let $\tilde{Q}$ be the complete inverse image of $Q$ in $F\left[X_{1}\right]$. Then $\tilde{P}$ is a prime of $F[X], \tilde{Q}$ is a prime of $F\left[X_{1}\right]$ and $\tilde{P} \cap F\left[X_{1}\right]=\bigcap_{x \in X} \tilde{Q}^{x}$. From this we see that $\tilde{Q}$ is a minimal covering prime of the intersection. Furthermore $X_{1}$ is an orbitally sound normal subgroup of finite index in $X$, by Proposition 3.2, so Proposition 3.5 applies.

Note that $X \supseteq Q^{t}$ implies that $\tilde{Q}^{\dagger}=Q^{t}$ and hence, by Lemma 3.4(ii), that

$$
b_{X}\left(\tilde{Q}^{\dagger}\right)=b_{X}\left(Q^{t}\right)=b_{\mathfrak{G}}\left(Q^{t}\right)=N^{T}
$$

We conclude from Proposition 3.5(i) applied to $F[X], X_{1}, \tilde{P}$, and $\tilde{Q}$ that there exists a prime ideal $\tilde{L}$ of $F\left[\nabla_{X}(N)\right]$ with $\tilde{L}^{X}=\tilde{P}$. Furthermore $\tilde{L}$ is unique up to conjugation by $\mathbf{N}_{X}(N)$ and $b_{X}\left(\tilde{L}^{\dagger}\right) \cap X_{1}=N$. It remains to translate this information back to $F^{t}[G]$.

Since $\mathfrak{G}=F^{*} X$ it is clear that $\mathbf{N}_{\mathfrak{G}}(N)=F^{*} \mathbf{N}_{X}(N)$ and hence that $\nabla_{\mathfrak{G}}(N)=$ $F^{\cdot} \nabla_{X}(N)$. Furthermore $\nabla_{X}(N) \supseteq Z$ so it follows that $\nabla_{X}(N)$ is the complete inverse image in $X$ of $\nabla_{G}^{t}(N)$ and in particular that $F\left[\nabla_{X}(N)\right]$ maps onto $F^{t}\left[\nabla_{G}^{t}(N)\right]$. If $\tilde{I}$ denotes the kernel of the homomorphism $F[X] \rightarrow F^{t}[G]$, then clearly $\tilde{I}=$ $(\tilde{I} \cap F[Z]) F[X]$ where $F[Z] /(\tilde{I} \cap F[Z]) \simeq F$. Since $\tilde{I} \subseteq \tilde{P}=\tilde{L}^{X} \subseteq \tilde{L} F[X]$ and $Z \subseteq \nabla_{X}(N)$, it follows that $\tilde{I} \cap F[Z] \subseteq \tilde{L}$. Thus $\tilde{L}$ is the complete inverse image of an ideal $L$ of $F^{t}\left[\nabla_{G}^{t}(N)\right]$ and certainly $L$ is prime. In addition, the formula

$$
\tilde{P}=\bigcap_{x \in X}(\tilde{L} F[X])^{x}=\tilde{L}^{X}
$$

maps into $F^{t}[G]$ to yield

$$
P=\bigcap_{x \in G}\left(L F^{t}[G]\right)^{x}=L^{G}
$$

and we have proved the existence of an appropriate prime $L$.

We now know that $L_{1}$, as chosen in the second paragraph of the proof, exists and we let $\tilde{L}_{1}$ denote its complete inverse image in $F\left[\nabla_{X}(N)\right]$. Since

$$
P=\bigcap_{x \in G}\left(L_{1} F^{t}[G]\right)^{x}=L_{1}^{G}
$$

it follows by taking complete inverse images that

$$
\tilde{P}=\bigcap_{x \in X}\left(\tilde{L}_{1} F[X]\right)^{x}=\tilde{L}_{1}^{X} .
$$


Thus by uniqueness in $F[X]$ we see that $\tilde{L}_{1}$ and $\tilde{L}$ are conjugate under $\mathbf{N}_{X}(N)$ and therefore $L_{1}$ and $L$ are conjugate under $\mathbf{N}_{\mathfrak{G}}(N)$. This proves the uniqueness of $L$.

Finally, by assumption, $X \supseteq L_{1}^{t}$ so $\tilde{L}_{1}^{\dagger}=L_{1}^{t}$ and $b_{\mathfrak{G}}\left(L_{1}^{t}\right)=b_{\mathfrak{G}}\left(\tilde{L}_{1}^{\dagger}\right)=b_{X}\left(\tilde{L}_{1}^{\dagger}\right)$ by Lemma $3.4\left(\right.$ ii). Since $\tilde{L}_{1}^{X}=\tilde{P}$, Proposition $3.5(\mathrm{i})$ yields $b_{X}\left(\tilde{L}_{1}^{\dagger}\right) \cap X_{1}=N$ and hence, since $X \cap \mathfrak{H}=X_{1}$, that $b_{\mathfrak{G}}\left(L_{1}^{t}\right) \cap \mathfrak{H}=N$. But $L$ and $L_{1}$ are conjugate under $\mathbf{N}_{\mathfrak{G}}(N)$ so since $\mathfrak{H} \triangleleft \mathfrak{G}$ we conclude that $b_{\mathfrak{G}}\left(L^{t}\right) \cap \mathfrak{H}=N$.

(ii) The argument here is of course similar and we freely use a good deal of the notation and observations of (i). Let $X$ be a finitely generated subgroup of $\mathfrak{G}$ with $F^{*} X=\mathfrak{G}$ and $X \supseteq L^{t} \supseteq N$. In addition, assume that $X \supseteq Q_{i}^{t}$ for the finitely many minimal covering primes $Q_{i}$ of $L^{G} \cap F^{t}[H]$. Clearly $N$ is a bounding, orbital subgroup of $X$ with $N \subseteq X_{1}$, the complete inverse image of $H=\operatorname{nio}^{2} G$ in $X$. Set $Z=F^{*} \cap X$ and let $\tilde{I}=(\tilde{I} \cap F[Z]) F[X]$ be the kernel of the epimorphism $F[X] \rightarrow F^{t}[G]$. Observe that $F\left[\nabla_{X}(N)\right]$ maps onto $F^{t}\left[\nabla_{G}^{t}(N)\right]$ so $\tilde{L}$, the complete inverse image of $L$, is a prime ideal of $F\left[\nabla_{X}(N)\right]$. By assumption $X \supseteq L^{t}$ so we have $L^{t}=\tilde{L}^{\dagger}$ and hence

$$
N=b_{\mathfrak{G}}\left(L^{t}\right) \cap \mathfrak{H}=b_{\mathfrak{G}}\left(\tilde{L}^{\dagger}\right) \cap \mathfrak{H} .
$$

But $b_{\mathfrak{B}}\left(\tilde{L}^{\dagger}\right)=b_{X}\left(\tilde{L}^{\dagger}\right)$ by Lemma $3.4(\mathrm{ii}), N \subseteq X$, and $\mathfrak{H} \cap X=X_{1}$ so this implies that $N=b_{X}\left(\tilde{L}^{\dagger}\right) \cap X_{1}$. We can now apply Proposition 3.5(ii) since $X_{1}$ is orbitally sound.

We conclude that $\tilde{P}=\tilde{L}^{X}$ is a prime ideal of $F[X]$ and that there exists a minimal covering prime $\tilde{Q}$ of $\tilde{P} \cap F\left[X_{1}\right]$ with $b_{X}\left(\tilde{Q}^{\dagger}\right)=N$. Since $\tilde{L} \supseteq \tilde{I} \cap F[Z]$ it follows that $\tilde{P} \supseteq \tilde{I}$ so that $\tilde{P}$ is the complete inverse image of a prime $P$ of $F^{t}[G]$. As we observed above, the formula $\tilde{P}=\tilde{L}^{X}$ maps to $P=L^{G}$. In addition, $\tilde{Q} \supseteq \tilde{P} \cap F\left[X_{1}\right] \supseteq \tilde{I} \cap F[Z]$ so $\tilde{Q}$ is the complete inverse image of a prime ideal $Q$ of $F^{t}[H]$. Since the formula $\tilde{P} \cap F\left[X_{1}\right]=\bigcap_{x \in X} \tilde{Q}^{x}$ maps to $P \cap F^{t}[H]=\bigcap_{x \in G} Q^{x}$ we see that $Q$ is a minimal covering prime of the latter intersection and hence of $L^{G} \cap F^{t}[H]$. Finally, by assumption, $X \supseteq Q^{t}$ so $\tilde{Q}^{\dagger}=Q^{t}$ and we conclude from Lemma 3.4(ii) that

$$
b_{\mathfrak{G}}\left(Q^{t}\right)=b_{\mathfrak{G}}\left(\tilde{Q}^{\dagger}\right)=b_{X}\left(\tilde{Q}^{\dagger}\right)=N
$$

This completes the proof.

We remark that the function of the above result, as with the work of [ $\mathbf{3}$ and $\mathbf{8}$ ], is to reduce the study of prime ideals to essentially commutative situations. Indeed let $F^{t}[G]$ and $N \subseteq \mathfrak{G}$ be given with $N \cap F^{*}=1$ and suppose for convenience that $G=\nabla_{G}^{t}(N)$. Let $I$ be the ideal of $F^{t}[G]$ generated by all $1-\alpha$ with $\alpha \in N$. Since $N \triangleleft \mathfrak{G}$ and $N \cap F^{*}=1$, it follows easily that $F^{t}[G] / I \simeq F^{t}[\tilde{G}]$ where $\tilde{G}=\mathfrak{G} / F^{*} N$ is a suitable homomorphic image of $G=\mathfrak{G} / F^{*}$. Furthermore the group of trivial units of $F^{t}[\tilde{G}]$ is $\tilde{\mathfrak{G}}=\mathfrak{G} / N$ and this is an f.c. group (that is $\tilde{\mathfrak{G}}=\Delta(\tilde{\mathfrak{G}})$ ) since $\mathfrak{G}=\nabla_{\mathfrak{G}}(N)$ by assumption.

Since $\tilde{G}$ is finitely generated, $\tilde{\mathfrak{G}}=F^{\bullet} \tilde{X}$ for some finitely generated subgroup $\tilde{X}$ and we conclude from [5, Lemma 4.1.5] that $|\tilde{\mathfrak{G}}: \mathbf{Z}(\tilde{\mathfrak{G}})|<\infty$ and that $\left|\tilde{\mathfrak{G}}^{\prime}\right|<\infty$. In particular if $\mathbf{Z}(\tilde{\mathfrak{G}}) / F^{\cdot}=\tilde{H} \subseteq \tilde{G}$, then $\tilde{H}$ is a central subgroup of $\tilde{G}$ of finite index and indeed $F^{t}[\tilde{H}]$ is a central subring of $F^{t}[\tilde{G}]$. Furthermore if $\tilde{T}$ is a torsion-free subgroup of $\tilde{H}$ of finite index, then since $F^{t}[\tilde{T}]$ is commutative it follows easily that $F^{t}[\tilde{T}]$ is isomorphic to the ordinary group algebra $F[\tilde{T}]$. In other words, $F^{t}[\tilde{G}]$ is a finite module over the well-understood central subring $F[\tilde{T}]$. 
Finally if $L$ is a prime ideal of $F^{t}[G]$ with $\left|L^{t}: N\right|<\infty$, then $L \supseteq I$ so $L$ is the complete inverse image of a prime ideal $\tilde{L}$ of the essentially commutative ring $F^{t}[\tilde{G}]$.

4. Conclusion. In this final section we combine our earlier results to indicate how the primes of $R * G$ can be described. As a consequence we obtain a bound on the classical Krull dimension, that is the prime length, of $R * G$.

LEMMA 4.1. Let $G$ be an arbitrary group acting as automorphisms on the right Noetherian ring $R$.

(i) $A G$-stable ideal $P$ of $R$ is $G$-prime if and only if there exists a $G$-orbital prime $Q$ of $R$ with $P=\bigcap_{x \in G} Q^{x}$. Furthermore this yields a one-to-one correspondence between the $G$-primes $P$ of $R$ and the $G$-conjugacy classes $\left\{Q^{x} \mid x \in G\right\}$ of $G$-orbital primes of $R$.

(ii) The $G$-prime length of $R$ is equal to the $G$-orbital prime length of $R$ and hence less than or equal to $\mathrm{cl}-\mathrm{K} \operatorname{dim} R$.

Proof. (i) The argument of Lemma 1.3 shows that if $P$ is a $G$-prime ideal of $R$ and $Q$ is a minimal covering prime, then $P=\bigcap_{x \in G} Q^{x}$ and $\left\{Q^{x} \mid x \in G\right\}$ is precisely the finite set of minimal covering primes. Thus $P$ determines $\left\{Q^{x}\right\}$ and certainly $\left\{Q^{x}\right\}$ determines $P$. Conversely let $Q$ be a $G$-orbital prime of $R$ and set $P=\bigcap_{x \in G} Q^{x}$ so that $P$ is $G$-stable. If $A$ and $B$ are $G$-stable ideals of $R$ with $A B \subseteq P \subseteq Q$ then, since $Q$ is prime, we have $A \subseteq Q$ or $B \subseteq Q$, say the latter. But $B$ is $G$-stable so $B \subseteq \bigcap_{x \in G} Q^{x}=P$ and thus $P$ is $G$-prime. Finally since $Q$ is $G$-orbital, $P=\bigcap_{x \in G} Q^{x}$ implies that $\left\{Q^{x}\right\}$ is the finite set of minimal covering primes and hence is determined by $P$.

(ii) Let $Q_{0}<Q_{1}<\cdots<Q_{n}$ be a chain of $G$-orbital primes of $R$ and set $P_{i}=\bigcap_{x \in G} Q_{i}^{x}$. Then each $P_{i}$ is $G$-prime and $P_{0}<P_{1}<\cdots<P_{n}$ by the uniqueness in (i). Conversely let $P_{0}<P_{1}<\cdots<P_{n}$ be a chain of $G$-prime ideals of $R$ and let $Q_{n}$ be a minimal covering prime of $P_{n}$. We choose $Q_{n-1}, Q_{n-2}, \ldots, Q_{0}$ in turn as follows. Given $Q_{i+1}$ we have $Q_{i+1} \supseteq P_{i+1}>P_{i}$ so $Q_{i+1}$ contains $Q_{i}$, a minimal covering prime of $P_{i}$. By the uniqueness in (i) we have $Q_{i+1}>Q_{i}$ and thus $Q_{0}<Q_{1}<\cdots<Q_{n}$ is a chain of $G$-orbital primes of $R$.

In particular in this Noetherian context the study of $G$-prime ideals is essentially equivalent to the study of $G$-orbital primes. For this reason we require the following cornpanion to Theorem 3.6.

PROPOSITION 4.2. Let $F^{t}[G]$ be a twisted group algebra of the polycyclic-byfinite group $G$ and let $\mathfrak{A}$ be a group acting as automorphisms on this ring, normalizing both $\mathfrak{G}$ and $F$. Set $H=\operatorname{nio}^{2} G$, let $\mathfrak{H}$ be the group of trivial units of $F^{t}[H] \subseteq F^{t}[G]$ and let $N$ be a bounding, orbital subgroup of $\mathfrak{G}$ with $N \subseteq \mathfrak{H}$ and $N \cap F^{*}=1$. If $L$ is a prime ideal of $F^{t}\left[\nabla_{G}^{t}(N)\right]$ with $b_{\mathfrak{G}}\left(L^{t}\right) \cap \mathfrak{H}=N$, then $P=L^{G}$ is an $\mathfrak{A}$-orbital prime ideal of $F^{t}[G]$ if and only if both $N$ and $L$ are $\mathfrak{A}$-orbital.

PROOF. If both $N$ and $L$ are $\mathfrak{A}$-orbital, then they are both stabilized by a subgroup $\mathfrak{B}$ of finite index in $\mathfrak{A}$. Clearly $\mathfrak{B}$ stabilizes the right ideal $L \cdot F^{t}[G]$ and hence the largest two-sided ideal contained in it, namely $L^{G}$.

Conversely let $P$ be $\mathfrak{A}$-orbital. Since $\mathfrak{A}$ normalizes $\mathfrak{G}$ and $F$, it acts on $G=\mathfrak{G} / F^{*}$ and hence normalizes $H=\operatorname{nio}^{2} G$ and therefore also $\mathfrak{H}$. If $P \cap F^{t}[H]=\bigcap_{x \in G} Q^{x}$ as in Theorem 3.6(i), then $\left\{Q^{x}\right\}$ is finite and uniquely determined by $P \cap F^{t}[H]$. 
It follows that there exists a subgroup $\mathfrak{B}$ of finite index in $\mathfrak{A}$ which stabilizes $P$ and all elements of the set $\left\{Q^{x}\right\}$. In particular $\mathfrak{B}$ stabilizes the prime $Q$ given by Theorem 3.6(ii) with $N=b_{\mathfrak{B}}\left(Q^{t}\right)$ and hence $\mathfrak{B}$ stabilizes $N$. This implies that $\mathfrak{B}$ stabilizes $\nabla_{G}^{t}(N)$ and therefore it stabilizes the finite set $\left\{L^{y} \mid y \in \mathbf{N}_{\mathfrak{B}}(N)\right\}$ of primes of $F^{t}\left[\nabla_{G}^{t}(N)\right]$ determined by $P$ and $N$. An appropriate subgroup of finite index in $\mathfrak{B}$ therefore stabilizes $L$.

It is now a simple matter to put this all together to describe the primes in these Noetherian crossed products. Thus let $R * G$ be given with $R$ right Noetherian and $G$ polycyclic-by-finite. If $P$ is a prime ideal of $R * G$ then, by Lemma 1.1, $A=P \cap R$ is a $G$-prime ideal of $R$. Furthermore $P \supseteq A * G$ and hence $P$ is the complete inverse image of a prime $\bar{P}$ of $(R * G) /(A * G) \simeq(R / A) * G$ with $\bar{P} \cap(R / A)=0$. Thus via this well-understood homomorphism, it suffices to assume that $P \cap R=0$ and hence that $R$ is $G$-prime.

Now Theorem 1.8 applies and we use its notation. In particular we let $Q$ be a minimal prime of $R$ with $H$ its stabilizer in $G$. Then $P=L^{G}$ where $L=P_{H}$ is a precisely determined prime ideal of $R * H$ with $L \cap R=Q$. Thus by passing to the subgroup $H$ of finite index in $G$ and moding out by $Q * H$ as above, it suffices to assume that $P \cap R=0$ and that $R$ is prime.

At this point Theorem 2.9 applies and we use its notation. Thus we extend $R * G$ to $S * G$ where $S=Q_{s}(R)$ and then

$$
T=\mathbf{C}_{S * G}(S)=F^{t}\left[G_{\text {inn }}\right]
$$

is a twisted group algebra of $G_{\text {inn }} \triangleleft G$ over the extended centroid $F=\mathbf{Z}(S)$. Furthermore $G$ acts as automorphisms on $F^{t}\left[G_{\text {inn }}\right]$ normalizing $F$ and the group of trivial units and $P$ is uniquely determined by a $G$-prime ideal $\tilde{P}$ of $T$. Specifically we have $P=(S * G) \tilde{P} \cap(R * G)$.

It remains to describe the $G$-prime ideals $P$ of $F^{t}[W]$ where $W=G_{\text {inn }}$ and of course the latter ring is right and left Noetherian by Lemma 1.2. Then by Lemma 4.1, $P=\bigcap_{x \in G} Q^{x}$ where $Q$ is a $G$-orbital prime of $F^{t}[W]$ and, by Theorem 3.6 and Proposition 4.2, $Q=L^{W}$ for a suitable $G$-orbital prime $L$ of $F^{t}\left[\nabla_{W}^{t}(N)\right]$. Here of course $N$ is a bounding, orbital subgroup of the group of trivial units of $F^{t}[W]$ with $N \cap F^{*}=1$. Since $Q=L^{W}=\bigcap_{w \in W}\left(L \cdot F^{t}[W]\right)^{w}$ and $W \subseteq G$ it follows that

$$
P=\bigcap_{x \in G}\left(L \cdot F^{t}[W]\right)^{x} .
$$

Finally as we observed at the end of the previous section, $L$ is the complete inverse image of a prime ideal $\bar{L}$ of a certain twisted group algebra $F^{t}[D]$, where $D$ is a homomorphic image of $\nabla_{W}^{t}(N)$. If $G_{1}$ is the stabilizer of $N$ in $G$, then $\left|G: G_{1}\right|<\infty, G_{1}$ acts on $F^{t}[D]$, and $\bar{L}$ is clearly $G_{1}$-orbital. Furthermore $D$ contains a torsion free abelian subgroup $T$ of finite index with $F^{t}[T]$ a central subring of $F^{t}[D]$ and $F^{t}[T] \simeq F[T]$. Thus a prime of a Noetherian crossed product $R * G$ eventually depends on a prime of an essentially commutative twisted group algebra $F^{t}[D]$ where $D$ is a group involved in $G$.

We consider a corollary on incomparability and prime length. If $G$ is polycyclicby-finite and if $1=G_{0} \triangleleft G_{1} \triangleleft \cdots \triangleleft G_{m}=G \triangleleft$ a subnormal series with quotients which are infinite cyclic or finite, then we recall that the number of infinite cyclic factors here is an invariant of $G$ called its Hirsch number and denoted by $h(G)$. It 
follows from $[\mathbf{9}, \S 2]$ that if $R$ is right Noetherian then

$$
\mathrm{K} \operatorname{dim} R * G \leq \mathrm{K} \operatorname{dim} R+h(G) .
$$

In particular since $R * G$ is also right Noetherian we have

$$
\operatorname{cl}-\mathrm{K} \operatorname{dim} R * G \leq \mathrm{K} \operatorname{dim} R * G \leq \mathrm{K} \operatorname{dim} R+h(G) .
$$

Among other things, the next result bounds the classical Krull dimension of $R * G$ in terms of cl-K $\operatorname{dim} R$ and $h(G)$. The bound is surely not sharp.

COROLlary 4.3. Let $R * G$ be given with $R$ right Noetherian and $G$ polycyclicby-finite with $h(G)=n$ If $P_{0}<P_{1}<\cdots<P_{n+1}$ are prime ideals of $R * G$, then $P_{0} \cap R<P_{n+1} \cap R$. Hence

$$
\operatorname{cl}-\mathrm{K} \operatorname{dim} R * G<(n+1)(1+\mathrm{cl}-\mathrm{K} \operatorname{dim} R) .
$$

PROOF. For the first part, let us suppose by way of contradiction that $P_{0} \cap R=$ $P_{n+1} \cap R$ so that all $P_{i} \cap R$ are equal. We can now follow the reductions as described above. Thus we can assume that $P_{0} \cap R=0$ and then, by dropping down to a subgroup of finite index which necessarily has the same Hirsch number, that $R$ is prime. This then gives rise to a chain of $G$-prime ideals $\tilde{P}_{0}<\tilde{P}_{1}<\cdots<\tilde{P}_{n+1}$ of $F^{t}\left[G_{\text {inn }}\right]$ and thus, by Lemma $4.1(\mathrm{ii}), n+1 \leq \operatorname{cl}-\mathrm{K} \operatorname{dim} F^{t}\left[G_{\text {inn }}\right]$. On the other hand, since $\mathrm{K} \operatorname{dim} F=0$ we have

$$
\operatorname{cl}-\mathrm{K} \operatorname{dim} F^{t}\left[G_{\mathrm{inn}}\right] \leq h\left(G_{\mathrm{inn}}\right) \leq h(G)=n
$$

so we have obtained the appropriate contradiction. We conclude therefore that $P_{0} \cap R<P_{n+1} \cap R$.

For the second part suppose that $P_{0}<P_{1}<\cdots<P_{m}$ is a strictly increasing chain of primes of $R * G$. Then by the above and Lemma 1.1(ii)

$$
P_{0} \cap R<P_{n+1} \cap R<P_{2(n+1)} \cap R<\cdots
$$

is a strictly increasing chain of $G$-primes of $R$. By applying Lemma 4.1(ii) to the group $\mathfrak{G}$ acting on $R$, we see that the latter chain can contain at most $1+\operatorname{cl}-\mathrm{K} \operatorname{dim} R$ terms. Hence $(n+1)(1+\mathrm{cl}-\mathrm{K} \operatorname{dim} R)>m$ as required.

This extends [1, Theorem 5.8] at least in the Noetherian situation. Presumably one should be able to replace the Hirsch number bound above by the smaller parameter defined in $[8]$ and called the plinth length. Roughly speaking the latter measures the number of infinite factors in a normal, rather than a subnormal, series for $G$. More precisely $p(G)$ can be described as the largest number of infinite factors $H_{i+1} / H_{i}$ in all series of the form

$$
1=H_{0} \subseteq H_{1} \subseteq \cdots \subseteq H_{m}=H \subseteq G
$$

where each $H_{i}$ is normal in $H$ and $|G: H|<\infty$ (see $[\mathbf{8}, \S 2.3]$ for basic properties). In particular $p(G) \leq h(G)$ and if $\left|G: G_{1}\right|<\infty$ then $p(G)=p\left(G_{1}\right)$. By [8, Theorem H1] the prime length of an ordinary group algebra $F[G]$ is equal to the plinth length of $G$. This can be lifed to

PROPOSITION 4.4. Let $F^{t}[G]$ be a twisted group algebra of the polycyclic-byfinite group $G$. Then $\mathrm{cl}-\mathrm{K} \operatorname{dim} F^{t}[G] \leq p(G)$.

PROOF. By reducing to a normal subgroup of finite index which changes neither the prime length $[\mathbf{1}$, Theorem 4.4] nor the plinth length, we can assume that $G$ is 
poly-(infinite cyclic). Let $X$ be a finitely generated subgroup of $\mathfrak{G}$ with $F^{\bullet} X=\mathfrak{G}$ so that $X$ is polycyclic-by-finite. To avoid confusion we set $Z=X \cap F^{*}$. Then $Z$ is a central subgroup of $X, X / Z=G$ and it follows easily that $p(X)=p(G)+h(Z)$.

Now the homomorphism $X \rightarrow \mathfrak{G}$ extends to an epimorphism $F[X] \rightarrow F^{t}[G]$ with kernel $I=(I \cap F[Z]) F[X]$ and $F[Z] /(I \cap F[Z]) \simeq F$. The latter implies that $I \cap F[Z]$ is conjugate via an automorphism of $F[Z]$ to the augmentation ideal and it then follows that there exists a chain of primes

$$
Q_{0}<Q_{1}<\cdots<Q_{h}=I \cap F[Z]
$$

of $F[Z]$ with $h=h(Z)$. Furthermore since $X / Z \simeq G$ is poly-(infinite cyclic) and $Z \subseteq \mathbf{Z}(X)$, we conclude that each $Q_{i} F[X]$ is a prime ideal of $F[X]$. In particular $I$ is a prime ideal and $F^{t}[G]$ is a prime ring.

Finally let $0=P_{0}<P_{1}<\cdots<P_{n}$ be a chain of prime ideals of $F^{t}[G]$ and let $\tilde{P}_{i}$ denote the complete inverse image of $P_{i}$ in $F[X]$. Since $\tilde{P}_{0}=I$, we obtain

$$
Q_{0} F[X]<Q_{1} F[X]<\cdots<Q_{h} F[X]=\tilde{P}_{0}<\tilde{P}_{1}<\cdots<\tilde{P}_{n}
$$

a chain of primes of $F[X]$ of length $n+h$. Hence by [8, Theorem H1]

$$
n+h \leq \mathrm{cl}-\mathrm{K} \operatorname{dim} F[X]=p(X)=p(G)+h
$$

so $n \leq p(G)$ as required.

We remark that equality need not hold in the above. For example, let $G=$ $\langle x\rangle \times\langle y\rangle$ be free abelian of rank 2 and let $F^{t}[G]$ be determined by the relation $\bar{x} \bar{y}=\lambda \bar{y} \bar{x}$ where $\lambda$ is an element of infinite multiplicative order in $F$. Then $F^{t}[G]$ is a simple ring and hence has prime length 0 . On the other hand, $p(G)=2$.

This result per se does not improve the bound in Corollary 4.3. The reason for this is that if $H \triangleleft G$ then it is quite possible to have $p(H)>p(G)$. Hence the argument fails when we drop from $G$ to $G_{\text {inn }} \triangleleft G$. To proceed further would require an extension of $[8$, Theorem $\mathrm{D}]$ to the situation where $\Gamma$ acts on $F[A]$ normalizing both $F$ and the group of trivial units. A little thought shows that such a result will not follow immediately from $[8$, Theorem D] by lifting and therefore this is best left for a later project.

\section{REFERENCES}

1. M. Lorenz and D. S. Passman, Prime ideals in crossed products of finite groups, Israel J. Math. 33 (1979), 89-132.

2. __ Addendum-Prime ideals in crossed products of finite groups, Israel J. Math. 35 (1980), 311-322.

3. __ Prime ideals in group algebras of polycyclic-by-finite groups, Proc. London Math. Soc. (3) 43 (1981), 520-543.

4. S. Montgomery and D. S. Passman, Crossed products over prime rings, Israel J. Math. 31 (1978), 224-256.

5. D. S. Passman, The algebraic structure of group rings, Wiley-Interscience, New York, 1977.

6. __ Computing the symmetric ring of quotients, J. Algebra (to appear).

7. __ Prime ideals in enveloping rings, Trans. Amer. Math. Soc. (to appear).

8. J. E. Roseblade, Prime ideals in group rings of polycyclic groups, Proc. London Math. Soc. (3) 36 (1978), 385-447.

9. P. F. Smith, On the dimension of group rings, Proc. London Math. Soc. (3) 25 (1972), 288-302.

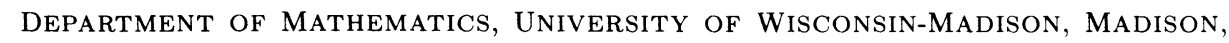
WISCONSIN 53706 